\title{
Antiviral treatment for treatment-naïve chronic hepatitis B: systematic review and network meta-analysis of randomized controlled trials
}

William W. L. Wong ${ }^{1,2,3^{*}}$ (B), Petros Pechivanoglou ${ }^{1,4}$, Josephine Wong ${ }^{1}$, Joanna M. Bielecki ${ }^{1}$, Alex Haines ${ }^{1}$, Aysegul Erman ${ }^{3}$, Yasmin Saeed ${ }^{3}$, Arcturus Phoon ${ }^{1}$, Mina Tadrous ${ }^{3,5}$, Mona Younis ${ }^{3}$, Noha Z. Rayad ${ }^{1,6}$,

Valeria Rac ${ }^{1,8,9,10}$, Harry L. A. Janssen ${ }^{7}$ and Murray D. Krahn ${ }^{1,3}$

\begin{abstract}
Background: Chronic hepatitis B (CHB) infection poses a significant burden to public health worldwide. Most cases are clinically silent until late in the disease course. The main goal of current therapy is to improve survival and quality of life by preventing disease progression to cirrhosis and liver failure, and consequently hepatocellular carcinoma development. The objective of this review is to provide a contemporary and comprehensive evaluation of the effectiveness of treatment options.
\end{abstract}

Methods: We performed a systematic review of peer-reviewed literature for randomized controlled trials involving treatment-naïve CHB adult population who received antiviral therapy. The endpoints were virologic response (VR), normalization of alanine aminotransferase (ALT norm), HBeAg loss, HBeAg seroconversion, and HBsAg loss for the HBeAg-positive population; and VR and ALT norm for the HBeAg-negative population. Network meta-analysis (NMA) was performed to synthesize evidence on the efficacy of treatment.

Results: Forty-two publications were selected. Twenty-three evaluated HBeAg-positive population, 13 evaluated HBeAg-negative population, and six evaluated both. We applied NMA to the efficacy outcomes of the two populations separately. Treatment strategies were ranked by the probability of achieving outcomes, and pairwise comparisons calculated from NMA were reported in odds ratios (OR). For HBeAg-positive population, tenofovir disoproxil fumarate (TDF) and tenofovir alafenamide (TAF) were the best for VR; OR vs adefovir $=14.29,95 \% \mathrm{Cl} 7.69-25$ and $12.5,95 \% \mathrm{Cl}$ 4.35-33.33 respectively. TAF was the best for achieving ALT norm (OR vs placebo $=12.5,95 \% \mathrm{Cl} 4.55-33.33$ ), HBeAg loss, and seroconversion (OR vs entecavir/TDF combination $=3.03,95 \% \mathrm{Cl} 1.04-8.84$ and 3.33, 95\% Cl 1.16-10 respectively). In the HBeAg-negative population, TDF and TAF were the best for VR (OR vs adefovir $=9.79,95 \% \mathrm{Cl} 2.38$ 42.7 and $11.71,95 \% \mathrm{Cl} 1.03-150.48$ respectively). Telbivudine and TAF were the best for ALT norm. Certain nucleos(t)ide combinations also had high probability of achieving positive outcomes.

Conclusions: Our results are consonant with current clinical guidelines and other evidence reviews. For both HBeAgpositive and HBeAg-negative populations, TDF and TAF are the most effective agents for virologic suppression, and TAF is effective across all outcomes.

Keywords: Chronic hepatitis B, Treatment-naïve, Systematic review, Network meta-analysis

\footnotetext{
* Correspondence: wwlwong@uwaterloo.ca

${ }^{1}$ Toronto Health Economics and Technology Assessment Collaborative

(THETA), University of Toronto and University Health Network, Toronto, ON,

Canada

${ }^{2}$ University of Waterloo, School of Pharmacy, Waterloo, ON, Canada

Full list of author information is available at the end of the article
}

(c) The Author(s). 2019 Open Access This article is distributed under the terms of the Creative Commons Attribution 4.0 International License (http://creativecommons.org/licenses/by/4.0/), which permits unrestricted use, distribution, and reproduction in any medium, provided you give appropriate credit to the original author(s) and the source, provide a link to the Creative Commons license, and indicate if changes were made. The Creative Commons Public Domain Dedication waiver (http://creativecommons.org/publicdomain/zero/1.0/) applies to the data made available in this article, unless otherwise stated. 


\section{Background}

The growing burden of chronic hepatitis B (CHB) infection poses a significant public health concern worldwide [1]. The main goal of CHB therapy is to improve survival and quality of life by preventing disease progression to cirrhosis and liver failure, and consequently hepatocellular carcinoma development. Additional goals of antiviral therapy are to prevent mother-to-child transmission, hepatitis $B$ reactivation, and the development of hepatitis $B$ virus-associated extrahepatic manifestations [2].

Interferon-alfa (IFN) was the first drug approved by the Food and Drug Administration (FDA) for the treatment of CHB in 1992. In 1998, lamivudine (LAM) was available as the first oral therapy for $\mathrm{CHB}$ [3]. However, LAM's effectiveness has been limited because of the development of antiviral resistance [4]. Over the years, other drugs have become available including pegylated interferon-alfa (PEG-IFN), adefovir (ADV), entecavir (ETV), telbivudine (TBV), tenofovir disoproxil fumarate (TDF), and recently tenofovir alafenamide (TAF) $[3,5]$. Among these drugs, in particular, IFN and PEG-IFN can produce long-term immune control without the need for long-term treatment in a small proportion of patients. For other treatments, lifelong administration may be required. Initiating therapy with these medications involves consideration of drug-specific trade-offs such as high and potentially lifelong medication costs, limited adherence, potential side effects, and the risk of antiviral resistance.

ETV, TDF, and TAF were approved for the treatment of CHB by FDA in 2005, 2008, and 2016 respectively [6]. They appeared to have a higher genetic barrier to resistance than the other nucleos(t)ides [4, 7]. Patients have also expressed the need for treatments with higher cure rates, higher barriers to resistance, better side effect profiles, and better coverage by drug plans [8].

In 2010, we conducted a systematic review and Bayesian meta-analysis to evaluate the relative efficacy of the first 12 months of CHB antiviral treatments [9]. We concluded that TDF and ETV were the most potent oral antiviral agents for hepatitis B e antigen (HBeAg)-positive patients, and TDF the most effective for HBeAg-negative patients. Nearly 10 years later, there are more data on long-term treatment and follow-up. Virological breakthrough has been observed with long-term therapy which may or may not be related to the emergence of genotypic resistance $[10,11]$. New adverse events have been reported notably drug effects on renal function and bone metabolism [12]. There were also new clinical trial data for the recently approved drug, TAF, and for new drug combinations.

The objective of this review was to provide a comprehensive and contemporary look at the effectiveness of $\mathrm{CHB}$ antiviral treatments. Our focus was on treatmentnaïve adult patients diagnosed with $\mathrm{HBeAg}$-positive or
HBeAg-negative CHB infection without co-infections and without decompensated cirrhosis, hepatocellular carcinoma, and liver transplantation. Patients were not further stratified by their baseline hepatitis B virus (HBV) DNA level, serum alanine aminotransferase (ALT) level, or HBV genotype even though these are recognized predictors of response to certain therapies. This was partly due to sample size considerations and partly because the review was intended to be a comprehensive overview.

\section{Methods}

We conducted a thorough exhaustive search of available literature and identified the evidence from randomized controlled trials (RCTs) investigating the comparative effectiveness among the CHB treatments (PEG-IFN, ADV, LAM, ETV, TBV, TDF, TAF as monotherapy or combination therapy) on treatment-naïve adult populations through a systematic review. We synthesized both direct and indirect evidence on efficacy for all possible comparisons using network meta-analysis (NMA).

The protocol for this systematic review was an extension of our earlier systematic review carried out under the sponsorship of the Ontario Drug Policy Research Network (ODPRN) and reported in 2015 [13].

\section{Endpoint definitions}

Since the eradication of HBV is not really achievable with the currently available treatments, treatment success is usually measured by surrogate endpoints using biomarkers which correlate with improvement in long-term clinical outcome. We included five widely accepted efficacy endpoints for the HBeAg-positive population: virologic response (VR), normalization of alanine aminotransferase level (ALT norm), HBeAg loss, HBeAg seroconversion, and hepatitis B surface antigen (HBsAg) loss; and two efficacy endpoints for the HBeAg-negative population: VR and ALT norm. HBsAg loss was originally included as one of the three efficacy endpoints for the HBeAg-negative population. It was subsequently excluded because the data collected were scarce and insufficient for the performance of network meta-analysis.

The primary objective of current treatment strategies is the induction of long-term suppression of HBV DNA levels [2]. Virologic response (VR) in our study was defined as the attainment of undetectable HBV DNA levels as determined by the polymerase chain reaction (PCR) test for the particular study. Threshold values for undetectable HBV DNA levels according to the method of techniques used for measurement were documented, as they could be a source of heterogeneity. Only studies where the threshold of detection was $\leq 200 \mathrm{IU} / \mathrm{ml}(1000$ copies $/ \mathrm{ml}$ ) were used in the analysis.

ALT normalization, which is achieved in most patients with long-term suppression of $\mathrm{HBV}$ replication, is an 
additional important endpoint [2]. ALT normalization in our review was defined as the reduction of ALT levels to below the upper limit of normal for that study.

The induction of HBeAg loss, with or without anti$\mathrm{HBe}$ seroconversion, in $\mathrm{HBeAg}$-positive $\mathrm{CHB}$ patients is a valuable endpoint, as it often represents a partial immune control of the CHB infection [2]. In our study, $\mathrm{HBeAg}$ loss was defined as achieving an undetectable level, using the threshold of detection used in each corresponding study; and HBeAg seroconversion was defined as undetectable $\mathrm{HBeAg}$ with the presence of antiHBeAg.

The optimal endpoint of HBsAg loss, with or without anti-HBs seroconversion, indicates profound suppression of HBV replication and viral protein expression. $\mathrm{HBsAg}$ loss was defined as achieving an undetectable level, using the threshold of detection used in each corresponding study.

\section{Systematic review procedures Literature search strategy}

This systematic review was designed in accordance with the Preferred Reporting Items for Systematic reviews and Metaanalyses (PRISMA statement) [14] and the protocol followed the PRISMA-P statement [15]. The literature search was performed by an information specialist using a peerreviewed search strategy. Published literature was identified by searching the following bibliographic databases: Ovid MEDLINE (1946-), Ovid MEDLINE In-Process; Ovid EMBASE (1974-); Cochrane Database of Systematic Reviews (CDSR), the Cochrane Central Register of Controlled Trials; and Web of Science: Science Citation Index Expanded (SCI-EXPANDED) (1900-) and Conference Proceedings Citation Index-Science (CPCI-S) (1990-). Our search started from the date of inception of each database until June 2017. Search terms included controlled vocabulary $(\mathrm{MeSH})$ and text-words in the following three concept areas: chronic hepatitis B (CHB), antiviral agents (pegylated interferon, lamivudine, adefovir, entecavir, telbivudine, tenofovir disoproxil fumarate, tenofovir alafenamide), and the following published and validated filter was applied: randomized control trials (RCTs) [16]. The search was limited to the English language. A detailed search strategy for MEDLINE (Ovid) and final search results are provided in Additional file 1.

\section{Study selection criteria}

This systematic review included RCTs that compared at least two $\mathrm{CHB}$ antiviral treatments or one treatment with placebo/no treatment in adult patients diagnosed with CHB. Studies were selected for inclusion in the systematic review based on the selection criteria presented in Table 1.
Table 1 Inclusion and exclusion criteria for the selection of randomized controlled trials

Inclusion criteria
- Adult patients ( $\geq 18$ years of age) diagnosed with HBeAg-positive and/
or HBeAg-negative chronic hepatitis B infection
- Treatment of interest: adefovir (ADV), entecavir (ETV), lamivudine (LAM),
pegylated interferon (PEG-IFN), telbivudine (TBV), tenofovir alafenamide
(TAF), tenofovir disoproxil fumarate (TDF); as monotherapy or as
combination therapy
- Comparators: treatment of interest versus placebo (PLA) or Treatment
of interest versus different Treatment of interest
- Outcomes (Efficacy):
1. Virologic response (VR)
2. Alanine aminotransferase normalization (ALT norm)
3. HBeAg loss
4. HBeAg seroconversion
5. HBsAg loss
- Study design: published, randomized, controlled interventional studies
Exclusion criteria
- Studies that did not meet the aforementioned selection criteria,
uncontrolled non-randomized studies, qualitative studies, observational
studies, duplicate publications, conference abstracts, narrative reviews,
and editorials were excluded
- Studies not conducted in English
- Studies on special populations: coinfections with HIV or other forms of
hepatitis, decompensated cirrhosis, hepatocellular carcinoma, liver
transplantation, and pregnancy
- Treatment duration shorter than 48 weeks (except for Interferon
- Treatment experienced population > 50\%
HBeAg hepatis B eantgen,

HBeAg hepatitis B e-antigen, $H B s A g$ hepatitis B s-antigen

\section{Study screening and quality assessment}

Two reviewers (AE and YS) independently screened the titles and abstracts of the identified studies to determine if they met the inclusion criteria, using a hierarchical screening method adapted from PRISMA. Subsequently, the full text of the eligible studies was assessed by the same reviewers independently. Disagreements between the two reviewers (AE and $\mathrm{YS}$ ) were resolved by discussion. Clinical experts were consulted and existing systematic reviews and meta-analyses were reviewed to identify any relevant studies that were missed. Each included RCT was assessed using the Cochrane risk of bias tool [17] on six validity domains: selection (sequence generation, allocation concealment), performance (blinding of participants and personnel), detection (blinding of outcome assessment), attrition, reporting, and other sources of bias.

\section{Data extraction}

Using a standard spreadsheet, the following data were extracted: (1) study design, (2) sample size, (3) patient 
characteristics, (4) treatment doses, (5) treatment and/or follow-up duration, and (6) efficacy outcome measures.

\section{Data analysis methods}

Network meta-analysis (NMA) methods were used to synthesize the evidence from the RCTs on relative effectiveness across the treatment options. Binomial likelihood functions were assumed for the binary events of interest, and a random effects model was used for all the outcomes. The NMA was conducted within a Bayesian framework using JAGS v.4.3.0 [18] and the R package "gemtc" [19]. Under this framework, the distribution of each parameter of interest (posterior distribution) was estimated through a Markov chain Monte Carlo simulation method. We ran three Markov chains and performed 70,000 simulations for each chain for each outcome, and we excluded the first 20,000 simulations to ensure that we selected only converged values. We further assessed the convergence for all models through the Gelman and Rubin diagnostic [20]. Vague priors were assumed throughout the model. Normal priors were assumed for treatment effectiveness parameters with a mean zero and a variance of 10,000 , while a uniform prior $\mathrm{U}(0,2)$ was assumed for the standard deviation of the heterogeneity parameter. Median estimates for the parameters of interest together with their 95\% credible intervals $(95 \% \mathrm{CrI})$ were constructed from the posterior distributions of the MCMC simulations. In addition, the probability of each treatment to be the best, second best, third best, etc. was presented for all treatment options using rankograms.

An important assumption underlying NMA is that of consistency between the direct and indirect evidence of relative efficacy. Inconsistency check evaluates the validity of a network meta-analysis by assessing the compatibility of direct and indirect evidence. If the results from direct evidence conflict with the results from indirect evidence then this highlights a problem of inconsistency in the network. In this case, one could argue that inconsistency in the network, that is the direct and indirect evidence, may not be compatible. Any presence of inconsistency in closed loops was assessed through the use of the node-split method [21]. The difference in effect size between the direct and indirect evidence was estimated and tested to see if the difference was significantly different from zero. Any $p$ values below 0.05 would indicate that the difference in effect size between direct and indirect evidence was statistically significant from zero and therefore inconsistency existed in the model. In the case where inconsistency was identified, the studies involved in the inconsistent loops of the network were reviewed to detect any clinical heterogeneity that might have caused the inconsistency.

Sensitivity analysis was performed to assess the impact of assumptions related to the type of model (fixed vs random) on the estimates of relative efficacy (Additional file 6).
NMA was conducted on five efficacy outcomes in the HBeAg-positive population: HBV DNA suppression (VR), ALT norm, HBeAg loss, HBeAg seroconversion, and HBsAg loss; and on two efficacy outcomes in the HBeAg-negative population: VR and ALT norm. Based on the NMA, we estimated the probability for each treatment strategy to achieve each outcome and the odds ratios (OR) of pairwise comparisons between treatment options. In addition, the probability of each treatment to be the best, second best, third best, etc. was presented for all treatment options using rankograms.

In the main NMA, we included studies with combination strategies as long as they were given to naïve $\mathrm{CHB}$ patients.

\section{Results}

Selection of randomized control trials

A total of 6319 studies were identified from the original literature (Additional file 1). After screening the titles and abstracts, 1040 potentially relevant publications were retrieved for full text review (kappa $=0.733$; good agreement). After full text review, 42 publications were selected for inclusion (kappa $=0.94$; very good agreement). Additional file 2 is a summary of the risk of bias assessment of the included studies and Additional file 3 describes the included study characteristics and the abbreviations for treatment strategies used in NMA. These 42 publications were for mono-infected treatment-naïve adult patients. Twenty-three publications evaluated HBeAg-positive population only [22-44] and 13 publications evaluated HBeAg-negative population only [45-57]. Six publications assessed both populations [58-63] and were included in both networks as they reported the outcomes separately for each population. Table 2 summarizes the included studies by interventions according to the HBeAg status. The total number of participants in each study ranged from 42 to 921 . The overall number of included participants was 12,885. Participant baseline characteristics in each study are summarized in Additional file 4.

As for the 998 articles excluded, the reasons for exclusion were lack of comparators or control (36\%); no outcomes of interest reported (28\%); duplicate papers, secondary analyses, modeling studies or abstracts (20\%); and studies on special subsets of patients such as co-infections, decompensated cirrhosis, hepatocellular carcinoma, hemodialysis, and transplant (5\%).

\section{Network meta-analyses for RCT: efficacy}

Initially, we have included eight monotherapies and 19 combination treatment strategies in the NMA for HBeAgpositive patients; and eight monotherapies and six combination treatment strategies in the NMA for HBeAg-negative patients. However, after conducting the inconsistency analysis, we identified evidence for significant inconsistencies (Additional file 5) in some of the network loops for the 
Table 2 Summary of included studies by treatments according to HBeAg status

\begin{tabular}{|c|c|c|c|c|}
\hline Treatments included in the network meta-analysis & Studies $(n)$ & $\mathrm{DB} R C T(n)$ & Patients $(n)$ & Publication years \\
\hline \multicolumn{5}{|l|}{ HBeAg-positive patients } \\
\hline Lamivudine (LAM) & 12 & 9 & 1925 & 1998-2015 \\
\hline Adefovir (ADV) & 6 & 3 & 652 & $2003-2015$ \\
\hline Telbivudine (TBV) & 4 & 3 & 692 & $2005-2014$ \\
\hline Entecavir (ETV) & 9 & 3 & 1127 & $2006-2017$ \\
\hline Tenofovir disoproxil fumarate (TDF) & 6 & 4 & 1065 & $2008-2017$ \\
\hline Tenofovir alafenamide (TAF) & 1 & 1 & 581 & 2016 \\
\hline Pegylated interferon (PEG-IFN) & 6 & 2 & 727 & $2005-2016$ \\
\hline Combination therapies & 14 & 4 & 1740 & 2005-2016 \\
\hline Placebo (PLA) & 4 & 3 & 330 & 1998-2014 \\
\hline Total & $29^{a}$ & $15^{\mathrm{a}}$ & 8839 & 1998-2017 \\
\hline \multicolumn{5}{|l|}{ HBeAg-negative patients } \\
\hline Lamivudine (LAM) & 9 & 8 & 1005 & 1999-2017 \\
\hline Adefovir (ADV) & 3 & 3 & 401 & $2003-2015$ \\
\hline Telbivudine (TBV) & 2 & 2 & 242 & 2009-2014 \\
\hline Entecavir (ETV) & 4 & 3 & 470 & $2006-2017$ \\
\hline Tenofovir disoproxil fumarate (TDF) & 3 & 3 & 544 & 2008-2016 \\
\hline Tenofovir alafenamide (TAF) & 1 & 1 & 285 & 2016 \\
\hline Pegylated interferon (PEG-IFN) & 6 & 2 & 375 & 2004-2016 \\
\hline Combination therapies & 7 & 2 & 525 & 2004-2016 \\
\hline Placebo (PLA) & 4 & 3 & 180 & 1999-2007 \\
\hline Total & $19^{a}$ & $13^{\mathrm{a}}$ & 4046 & 1999-2017 \\
\hline
\end{tabular}

HBeAg hepatitis B e antigen, $n$ number, DB RCT double-blind randomized controlled trial

anique studies

efficacy results. After review of the relevant studies for the loops with evidence for inconsistency, we determined that the inconsistencies were likely to have originated from the fact that the included treatment PEG-IFN had a different mechanism of action from the oral nucleos(t)ides. Among these studies, PEG-IFN was used in different dosages and schedules and was combined in different order with oral nucleos(t)ides in combination therapy. On the basis of the clinical heterogeneity, we decided to further exclude studies that included the PEG-IFN treatment.

In the final NMA, we included seven monotherapies and six combination treatment strategies in the NMA for HBeAg-positive patients; and seven monotherapies and one combination treatment strategy in the NMA for HBeAg-negative patients. The evidence networks are presented in Fig. 1a, b for virologic response, Fig. 2a, b for ALT normalization, Fig. 3a, b for HBeAg loss and seroconversion respectively, and Fig. 4 for HBsAg loss. In these evidence networks, the size of the circles corresponds to the number of patients exposed to a treatment and the thickness of the connecting lines corresponds to the number of studies comparing the treatments directly. The relative efficacy in terms of odds ratios and $95 \%$ credible intervals of pairwise comparisons for each outcome are presented in Tables 3 and 4 . The probability of each treatment being the 1st, 2nd, 3rd, etc. are provided in the form of rankograms in Fig. 5a, b for the e-positive and e-negative populations respectively. We also compared the variances across all the node split models for each outcome against the consistent model. There were no significant fluctuations of the variance and its confidence interval across all models, with large overlap across the confidence intervals. This finding indicated limited evidence of inconsistency.

\section{$H B e A g$ positive patients: viral suppression and ALT normalization}

In pairwise comparisons across all drugs (Table 3), TDF had statistically significantly higher odds of achieving viral suppression than the rest except for ETVTDF and TAF, e.g., ETV vs TDF, OR = 0.46, 95\% CrI 0.25-0.86; TAF vs $\mathrm{TDF}, \mathrm{OR}=0.88,95 \mathrm{CrI} 0.38-1.99$. TDF had a probability of $43 \%$ being the best treatment for achieving virologic response, followed by the combination strategy ETVTDF (29\%) and TAF (26\%) (Fig. 5a). For ALT normalization, 


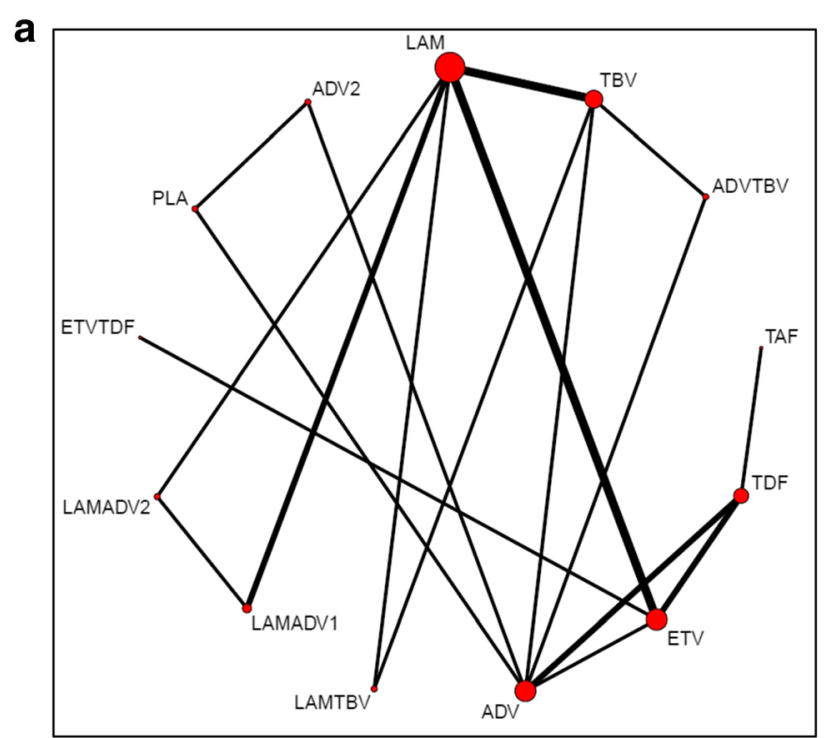

b

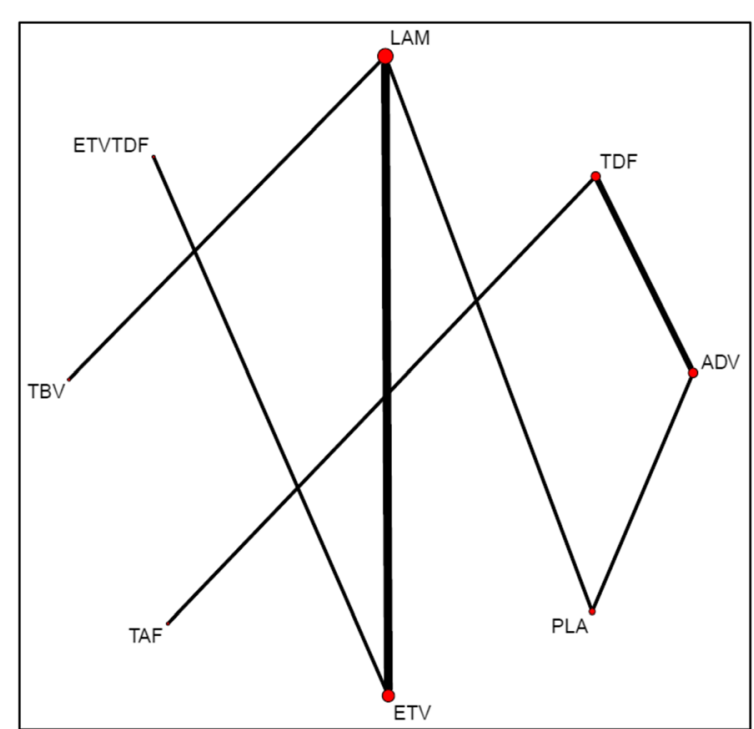

Fig. 1 a Evidence network for virologic response for HBeAg-positive patients. b Evidence network for virologic response for HBeAg-negative patients. ADV adefovir $10 \mathrm{mg}$ daily, ADV2 adefovir $30 \mathrm{mg}$ daily, ETV entecavir, LAM lamivudine, TBV telbivudine, PLA placebo, TAF tenofovir alafenamide, TDF tenofovir disoproxil fumarate; ADVTBV, ETVTDF, LAMADV1, LAMADV2, LAMTBV, and ETVTDF code for different combinations of antiviral agents with full details in Additional file 3

pairwise comparisons (Table 3) showed that no one drug was consistently superior. The combination LAMADV2 was significantly better than LAM $(\mathrm{OR}=0.35,95 \% \mathrm{Crl}$ 0.16-0.76), ADV (OR =0.33, 95\% Crl 0.13-0.96), and ETVTDF $(\mathrm{OR}=0.25,95 \%$ CrI 0.08-0.86). LAMADV2 had a probability of $52 \%$ being the best treatment followed by TAF (16\%) (Fig. 5a).
HBeAg-positive patients: $\mathrm{HBeAg}$ loss, $\mathrm{HBeAg}$ seroconversion, and $\mathrm{HBsAg}$ loss

For HBeAg seroconversion, pairwise comparison (Table 4) showed that LAMADV was significantly better than LAMADV1 $(\mathrm{OR}=3.59,95 \%$ CrI 1.22-10.74), LAMADV2 $(\mathrm{OR}=4.04,95 \% \mathrm{CrI} 1.31-13.28)$, and LAMTBV $(\mathrm{OR}=5.68,95 \% \mathrm{CrI} 1.43-24.18)$; and $\mathrm{TAF}$
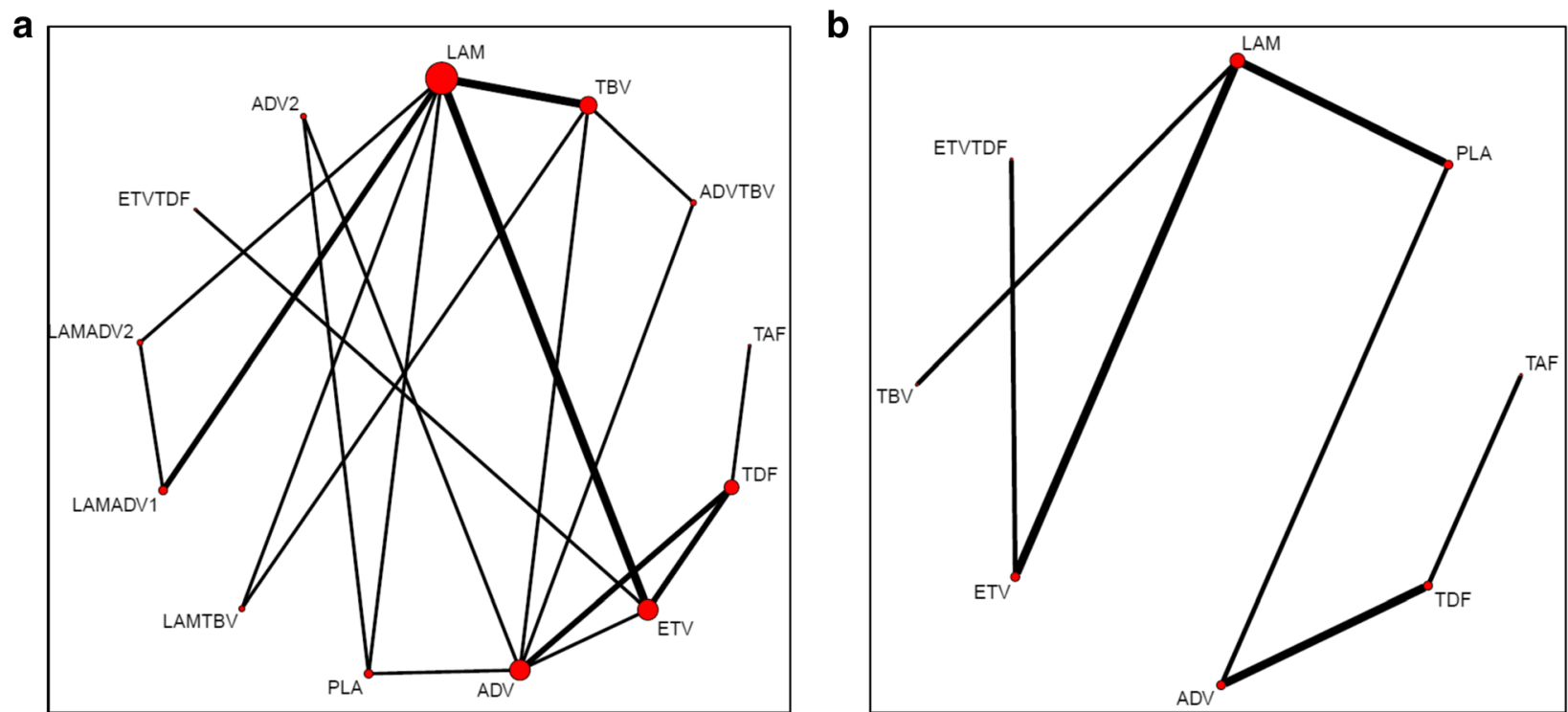

Fig. 2 a Evidence network for ALT normalization for HBeAg-positive patients. b Evidence network for ALT normalization for HBeAg-negative patients. ADV adefovir $10 \mathrm{mg}$ daily, ADV2 adefovir $30 \mathrm{mg}$ daily, ETV entecavir, LAM lamivudine, TBV telbivudine, PLA placebo, TAF tenofovir alafenamide, TDF tenofovir disoproxil fumarate; ADVTBV, ETVTDF, LAMADV1, LAMADV2, LAMTBV, and ETVTDF code for different combinations of antiviral agents with full details in Additional file 3 

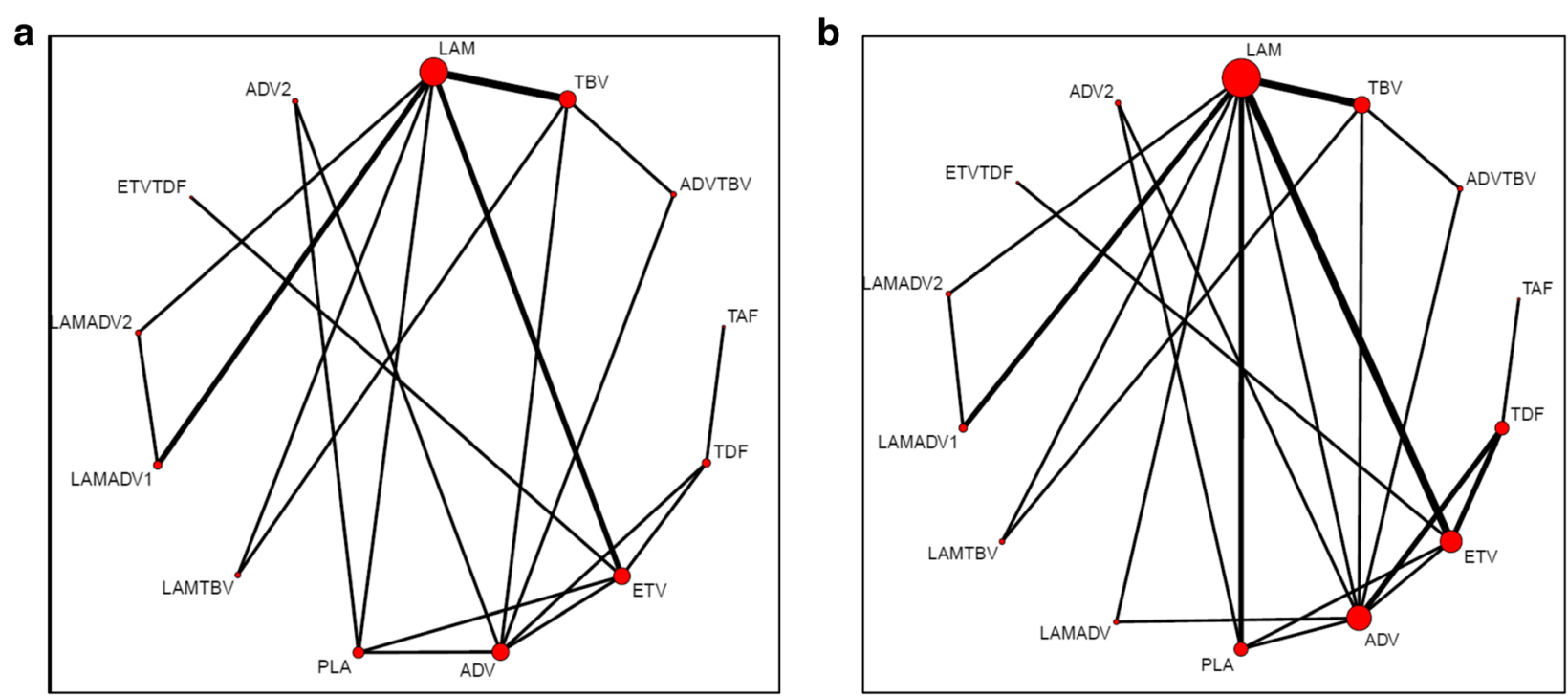

Fig. 3 a Evidence network for HBeAg loss for HBeAg-positive patients. b Evidence network for HBeAg seroconversion for HBeAg-positive patients, ADV adefovir $10 \mathrm{mg}$ daily, ADV2 adefovir $30 \mathrm{mg}$ daily, ETV entecavir, LAM lamivudine, TBV telbivudine, PLA placebo, TAF tenofovir alafenamide, TDF tenofovir disoproxil fumarate; ADVTBV, ETVTDF, LAMADV, LAMADV1, LAMADV2, and LAMTBV code for different combinations of antiviral agents with full details in Additional file 3

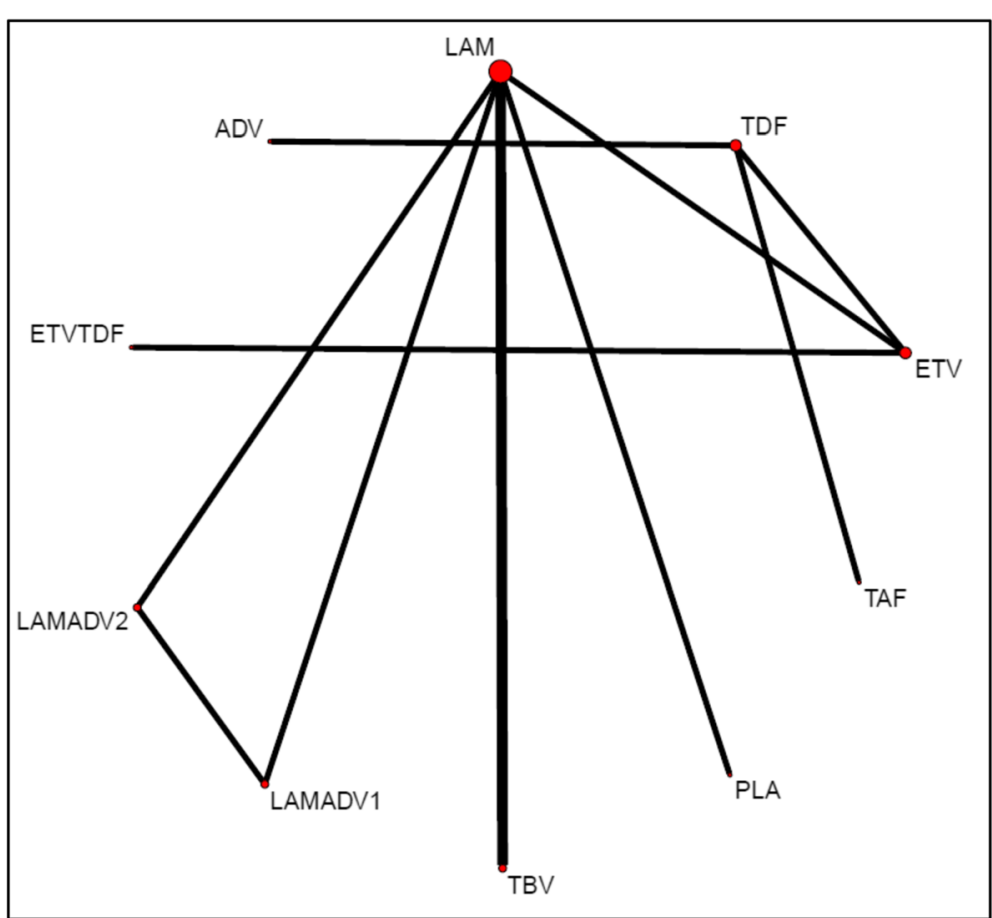

Fig. 4 Evidence network for HBsAg loss for HBeAg-positive patients. ADV adefovir $10 \mathrm{mg}$ daily, ETV entecavir, LAM lamivudine, TBV telbivudine, PLA placebo, TAF tenofovir alafenamide, TDF tenofovir disoproxil fumarate; ETVTDF, LAMADV1, and LAMADV2 code for different combinations of antiviral agents with full details in Additional file 3 
Table 3 Relative effects on virologic response and ALT normalization of all pairs of interventions in odds ratios (95\% credible intervals) as calculated from the network meta-analyses using random effects models

Virologic response: $\mathrm{HBeAg}$-positive population in the left lower triangle ad $\mathrm{HBeAg}$-negative population in the right upper triangle

\begin{tabular}{|c|c|c|c|c|c|c|c|c|c|c|c|c|}
\hline \multicolumn{13}{|c|}{ Between study heterogeneity HBeAg-negative: $0.69(0.09-1.77)$} \\
\hline ADV & & & $\begin{array}{l}2.35(0.03, \\
112.8)\end{array}$ & $\begin{array}{l}1.97(0.01, \\
177.67)\end{array}$ & $\begin{array}{l}0.35(0, \\
12.36)\end{array}$ & & & & $\begin{array}{l}1.32(0.01, \\
66.31)\end{array}$ & $\begin{array}{l}0.01(0, \\
0.13)\end{array}$ & $\begin{array}{l}11.71 \\
(1.03, \\
150.48) \\
\end{array}$ & $\begin{array}{l}9.79(2.38, \\
42.7)\end{array}$ \\
\hline $\begin{array}{l}0.43(0.18 \\
1.02)\end{array}$ & ADV2 & & & & & & & & & & & \\
\hline $\begin{array}{l}0.4(0.14, \\
1.15)\end{array}$ & $\begin{array}{l}0.94(0.24, \\
3.74)\end{array}$ & ADVTBV & & & & & & & & & & \\
\hline $\begin{array}{l}0.15(0.08 \\
0.31)\end{array}$ & $\begin{array}{l}0.36(0.12, \\
1.12)\end{array}$ & $\begin{array}{l}0.39(0.13, \\
1.15)\end{array}$ & ETV & $\begin{array}{l}0.86(0.09 \\
7.93)\end{array}$ & $\begin{array}{l}0.15(0.04, \\
0.45)\end{array}$ & & & & $\begin{array}{l}0.57(0.07 \\
3.75)\end{array}$ & $0(0,0.04)$ & $\begin{array}{l}4.99(0.05 \\
796.14)\end{array}$ & $\begin{array}{l}4.18(0.07, \\
447.24)\end{array}$ \\
\hline $\begin{array}{l}0.09(0.03 \\
0.28)\end{array}$ & $\begin{array}{l}0.2(0.05, \\
0.87)\end{array}$ & $\begin{array}{l}0.22(0.05, \\
0.92)\end{array}$ & $\begin{array}{l}0.56(0.22, \\
1.43)\end{array}$ & ETVTDF & $\begin{array}{l}0.17(0.01, \\
1.96)\end{array}$ & & & & $\begin{array}{l}0.66(0.03, \\
12.16)\end{array}$ & $0(0,0.12)$ & $\begin{array}{l}5.96(0.03 \\
1464.68)\end{array}$ & $\begin{array}{l}5.04(0.05, \\
802.29)\end{array}$ \\
\hline $\begin{array}{l}0.46(0.22, \\
1.07)\end{array}$ & $\begin{array}{l}1.09(0.35, \\
3.69)\end{array}$ & $\begin{array}{l}1.16(0.4 \\
3.61)\end{array}$ & $\begin{array}{l}3(1.92, \\
5.33)\end{array}$ & $\begin{array}{l}5.38(1.93, \\
16.65)\end{array}$ & LAM & & & & $\begin{array}{l}3.76(0.88, \\
18.74)\end{array}$ & $\begin{array}{l}0.03(0, \\
0.25)\end{array}$ & $\begin{array}{l}33.96 \\
(0.46, \\
5084.16)\end{array}$ & $\begin{array}{l}28.01 \\
(0.64, \\
2843.24)\end{array}$ \\
\hline $\begin{array}{l}0.21(0.08, \\
0.64)\end{array}$ & $\begin{array}{l}0.5(0.13, \\
2.05)\end{array}$ & $\begin{array}{l}0.54(0.15, \\
2.05)\end{array}$ & $\begin{array}{l}1.39(0.6 \\
3.48)\end{array}$ & $\begin{array}{l}2.48(0.71 \text {, } \\
9.56)\end{array}$ & $\begin{array}{l}0.46(0.22, \\
0.93)\end{array}$ & $\begin{array}{l}\text { LAMAD } \\
\text { V1 }\end{array}$ & & & & & & \\
\hline $\begin{array}{l}0.26(0.09 \\
0.88)\end{array}$ & $\begin{array}{l}0.61(0.15 \\
2.78)\end{array}$ & $\begin{array}{l}0.66(0.17, \\
2.69)\end{array}$ & $\begin{array}{l}1.7(0.67, \\
4.91)\end{array}$ & $\begin{array}{l}3.03(0.82 \\
13.11)\end{array}$ & $\begin{array}{l}0.56(0.24, \\
1.35)\end{array}$ & $\begin{array}{l}1.23(0.52, \\
2.9)\end{array}$ & $\begin{array}{l}\text { LAMAD } \\
\text { V2 }\end{array}$ & & & & & \\
\hline $\begin{array}{l}0.31(0.09 \\
1.16)\end{array}$ & $\begin{array}{l}0.73(0.16, \\
3.59)\end{array}$ & $\begin{array}{l}0.78(0.18, \\
3.44)\end{array}$ & $\begin{array}{l}2.04(0.64, \\
6.68)\end{array}$ & $\begin{array}{l}3.63(0.81, \\
16.65)\end{array}$ & $\begin{array}{l}0.67(0.22 \text {, } \\
1.95)\end{array}$ & $\begin{array}{l}1.46(0.4, \\
5.29)\end{array}$ & $\begin{array}{l}1.19(0.3 \\
4.74)\end{array}$ & LAMTBV & & & & \\
\hline $\begin{array}{l}0.22(0.1, \\
0.51)\end{array}$ & $\begin{array}{l}0.52(0.16 \\
1.76)\end{array}$ & $\begin{array}{l}0.55(0.2, \\
1.6)\end{array}$ & $\begin{array}{l}1.42(0.77 \\
2.9)\end{array}$ & $\begin{array}{l}2.54(0.83 \\
8.46)\end{array}$ & $\begin{array}{l}0.47(0.28, \\
0.77)\end{array}$ & $\begin{array}{l}1.03(0.43 \\
2.48)\end{array}$ & $\begin{array}{l}0.84(0.31, \\
2.26)\end{array}$ & $\begin{array}{l}0.7(0.25 \\
2.05)\end{array}$ & TBV & $\begin{array}{l}0.01(0, \\
0.1)\end{array}$ & $\begin{array}{l}8.95(0.09 \\
1619.77)\end{array}$ & $\begin{array}{l}7.45(0.12, \\
881.78)\end{array}$ \\
\hline $\begin{array}{l}67.72 \\
(9.98, \\
1980.93)\end{array}$ & $\begin{array}{l}157.85 \\
(23.8, \\
4603.65) \\
\end{array}$ & $\begin{array}{l}172.26 \\
(18.48, \\
5538)\end{array}$ & $\begin{array}{l}443.57 \\
(55.64, \\
13333.24)\end{array}$ & $\begin{array}{l}805.74 \\
(79.66, \\
25985.81)\end{array}$ & $\begin{array}{l}146.23 \\
(17.41, \\
4463.24)\end{array}$ & $\begin{array}{l}322.1 \\
(33.53 \\
10176.83) \\
\end{array}$ & $\begin{array}{l}264.97 \\
(25.83, \\
8576.92)\end{array}$ & $\begin{array}{l}223.05 \\
(20.86, \\
7397.65) \\
\end{array}$ & $\begin{array}{l}309.27 \\
(36.57 \\
9478.9)\end{array}$ & PLA & $\begin{array}{l}1213.15 \\
(35.51, \\
116539.09 \\
)\end{array}$ & $\begin{array}{l}1006.66 \\
(53.81, \\
53939.53)\end{array}$ \\
\hline $\begin{array}{l}0.08(0.03, \\
0.23)\end{array}$ & $\begin{array}{l}0.19(0.05, \\
0.75)\end{array}$ & $\begin{array}{l}0.2(0.05 \\
0.82)\end{array}$ & $\begin{array}{l}0.52(0.19 \\
1.49)\end{array}$ & $\begin{array}{l}0.93(0.23, \\
3.84)\end{array}$ & $\begin{array}{l}0.17(0.05, \\
0.51)\end{array}$ & $\begin{array}{l}0.38(0.1, \\
1.35)\end{array}$ & $\begin{array}{l}0.31(0.07, \\
1.2)\end{array}$ & $\begin{array}{l}0.26(0.06, \\
1.16)\end{array}$ & $\begin{array}{l}0.37(0.11, \\
1.14)\end{array}$ & $0(0,0.01)$ & TAF & $\begin{array}{l}0.84(0.38, \\
1.94)\end{array}$ \\
\hline $\begin{array}{l}0.07(0.04 \\
0.13)\end{array}$ & $\begin{array}{l}0.17(0.06, \\
0.49)\end{array}$ & $\begin{array}{l}0.18(0.06, \\
0.55)\end{array}$ & $\begin{array}{l}0.46(0.25, \\
0.86)\end{array}$ & $\begin{array}{l}0.82(0.27, \\
2.53)\end{array}$ & $\begin{array}{l}0.15(0.07, \\
0.31)\end{array}$ & $\begin{array}{l}0.33(0.11, \\
0.91)\end{array}$ & $\begin{array}{l}0.27(0.08, \\
0.81)\end{array}$ & $\begin{array}{l}0.23(0.06, \\
0.81)\end{array}$ & $\begin{array}{l}0.32(0.14 \\
0.7 I)\end{array}$ & $0(0,0.01)$ & $\begin{array}{l}0.88(0.38, \\
1.99)\end{array}$ & TDF \\
\hline
\end{tabular}

ALT normalization: HBeAg-positive population in the left lower triangle and $\mathrm{HBeAg}$-negative population in the right upper triangle

\begin{tabular}{|c|c|c|c|c|c|c|c|c|c|c|c|c|}
\hline \multicolumn{13}{|c|}{ Between study heterogeneity HBeAg-negative: $0.72(0.04-1.84)$} \\
\hline ADV & & & $\begin{array}{l}3.87(0.19 \text {, } \\
92.73)\end{array}$ & \begin{tabular}{|l}
$3.31(0.07$ \\
$159.16)$ \\
\end{tabular} & $\begin{array}{l}1.96(0.12, \\
26.63)\end{array}$ & & & & \begin{tabular}{|l}
$3.63(0.18$ \\
$80.15)$ \\
\end{tabular} & $\begin{array}{l}0.15(0.02 \\
1.2)\end{array}$ & $\begin{array}{l}2.2(0.17 \\
26.82)\end{array}$ & $\begin{array}{l}1.03(0.24, \\
4.55) \\
\end{array}$ \\
\hline $\begin{array}{l}0.81(0.42, \\
1.68) \\
\end{array}$ & ADV2 & & & & & & & & & & & \\
\hline $\begin{array}{l}0.65(0.2, \\
2.03)\end{array}$ & $\begin{array}{l}0.8(0.21 \\
2.98)\end{array}$ & ADVTBV & & & & & & & & & & \\
\hline $\begin{array}{l}0.54(0.3, \\
0.98)\end{array}$ & $\begin{array}{l}0.66(0.28, \\
1.56) \\
\end{array}$ & \begin{tabular}{|l|}
$0.83(0.25$ \\
$2.83)$ \\
\end{tabular} & ETV & \begin{tabular}{|l|}
$0.85(0.09$ \\
$7.52)$ \\
\end{tabular} & $\begin{array}{l}0.53(0.09, \\
1.98) \\
\end{array}$ & & & & $\begin{array}{l}0.95(0.1 \\
8.13)\end{array}$ & $\begin{array}{l}0.04(0, \\
0.34)\end{array}$ & $\begin{array}{l}0.57(0.01, \\
27.68)\end{array}$ & $\begin{array}{l}0.27(0.01 \\
7.37)\end{array}$ \\
\hline $\begin{array}{l}1.34(0.5, \\
3.69)\end{array}$ & $\begin{array}{l}1.64(0.51, \\
5.34)\end{array}$ & $\begin{array}{l}2.06(0.48, \\
8.96)\end{array}$ & $\begin{array}{l}2.49(1.11, \\
5.66)\end{array}$ & ETVTDF & \begin{tabular}{|l}
$0.6(0.03$ \\
$7.19)$ \\
\end{tabular} & & & & $\begin{array}{l}1.12(0.05, \\
23.27)\end{array}$ & $\begin{array}{l}0.05(0, \\
1.02)\end{array}$ & $\begin{array}{l}0.67(0.01, \\
58.29)\end{array}$ & $\begin{array}{l}0.31(0.01, \\
17.71)\end{array}$ \\
\hline $\begin{array}{l}0.96(0.53 \\
1.93)\end{array}$ & $\begin{array}{l}1.18(0.52 \\
2.93)\end{array}$ & $\begin{array}{l}1.48(0.48 \\
5.07)\end{array}$ & $\begin{array}{l}1.78(1.21, \\
2.95)\end{array}$ & \begin{tabular}{|l}
$0.72(0.3$, \\
$1.87)$ \\
\end{tabular} & LAM & & & & $\begin{array}{l}1.82(0.45 \\
9.92)\end{array}$ & $\begin{array}{l}0.08(0.02, \\
0.45)\end{array}$ & $\begin{array}{l}1.1(0.03, \\
48.24)\end{array}$ & $\begin{array}{l}0.52(0.03 \\
12.03)\end{array}$ \\
\hline $\begin{array}{l}0.43(0.18 \\
1.12) \\
\end{array}$ & \begin{tabular}{|l|}
$0.53(0.19$ \\
$1.61)$ \\
\end{tabular} & \begin{tabular}{|l|}
$0.66(0.19$ \\
$2.7)$ \\
\end{tabular} & \begin{tabular}{|l}
$0.8(0.39$ \\
$1.84)$ \\
\end{tabular} & \begin{tabular}{|l|}
$0.32(0.11$, \\
$1.03)$ \\
\end{tabular} & $\begin{array}{l}0.44(0.24, \\
0.84) \\
\end{array}$ & LAMADV1 & & & & & & \\
\hline $\begin{array}{l}0.33(0.13 \\
0.96)\end{array}$ & $\begin{array}{l}0.41(0.13 \\
1.35)\end{array}$ & \begin{tabular}{|l|}
$0.51(0.13$ \\
$2.22)$ \\
\end{tabular} & \begin{tabular}{|l|}
$0.62(0.26$, \\
$1.59)$ \\
\end{tabular} & \begin{tabular}{|l|}
$0.25(0.08$ \\
$0.86)$ \\
\end{tabular} & $\begin{array}{l}0.35(0.16 \\
0.76)\end{array}$ & \begin{tabular}{|l|}
$0.77(0.34$ \\
$1.76)$ \\
\end{tabular} & LAMADV2 & & & & & \\
\hline $\begin{array}{l}0.76(0.21, \\
2.73)\end{array}$ & $\begin{array}{l}0.92(0.23, \\
3.78)\end{array}$ & \begin{tabular}{|l}
$1.15(0.24$, \\
$5.9)$
\end{tabular} & $\begin{array}{l}1.39(0.43, \\
4.77) \\
\end{array}$ & \begin{tabular}{|l}
$0.56(0.14$ \\
$2.42)$ \\
\end{tabular} & $\begin{array}{l}0.77(0.25, \\
2.41)\end{array}$ & $\begin{array}{l}1.73(0.47, \\
6.36) \\
\end{array}$ & \begin{tabular}{|l}
$2.25(0.56$, \\
$8.87)$ \\
\end{tabular} & LAMTBV & & & & \\
\hline $\begin{array}{l}0.63(0.32 \\
1.32)\end{array}$ & $\begin{array}{l}0.77(0.31, \\
2)\end{array}$ & \begin{tabular}{|l|}
$0.96(0.31$, \\
$3.23)$ \\
\end{tabular} & $\begin{array}{l}1.16(0.67, \\
2.18) \\
\end{array}$ & \begin{tabular}{|l}
$0.47(0.17$ \\
$1.31)$ \\
\end{tabular} & $\begin{array}{l}0.65(0.41, \\
0.98)\end{array}$ & \begin{tabular}{|l}
$1.46(0.66$, \\
$3.04)$
\end{tabular} & $\begin{array}{l}1.88(0.76, \\
4.61)\end{array}$ & \begin{tabular}{|l}
$0.84(0.27$ \\
$2.56)$ \\
\end{tabular} & TBV & $\begin{array}{l}0.04(0, \\
0.38)\end{array}$ & $\begin{array}{l}0.6(0.01 \\
30.93) \\
\end{array}$ & $\begin{array}{l}0.28(0.01, \\
7.9) \\
\end{array}$ \\
\hline $\begin{array}{l}5.94(3.24, \\
12.04)\end{array}$ & $\begin{array}{l}7.29(3.61, \\
15.92)\end{array}$ & $\begin{array}{l}9.12(2.63, \\
33.35)\end{array}$ & $\begin{array}{l}11.03(5.48, \\
24.01)\end{array}$ & $\begin{array}{l}4.43(1.52, \\
13.57) \\
\end{array}$ & $\begin{array}{l}6.15(3.04, \\
12.32) \\
\end{array}$ & $\begin{array}{l}13.88(5.25, \\
34.65)\end{array}$ & $\begin{array}{l}17.8(6.12, \\
51.02)\end{array}$ & $\begin{array}{l}7.97(2.13, \\
29.19)\end{array}$ & $\begin{array}{l}9.48(4.32, \\
20.99)\end{array}$ & PLA & $\begin{array}{l}14.6(0.55 \\
373.8)\end{array}$ & $\begin{array}{l}6.86(0.54 \\
85.5) \\
\end{array}$ \\
\hline $\begin{array}{l}0.48(0.21 \\
1.16)\end{array}$ & $\begin{array}{l}0.59(0.21 \\
1.73)\end{array}$ & $\begin{array}{l}0.74(0.19, \\
3.03) \\
\end{array}$ & $\begin{array}{l}0.9(0.39, \\
2.14) \\
\end{array}$ & $\begin{array}{l}0.36(0.11 \\
1.17)\end{array}$ & $\begin{array}{l}0.5(0.19 \\
1.21) \\
\end{array}$ & $\begin{array}{l}1.13(0.35 \\
3.23)\end{array}$ & $\begin{array}{l}1.45(0.42, \\
4.75)\end{array}$ & $\begin{array}{l}0.65(0.15 \\
2.68) \\
\end{array}$ & $\begin{array}{l}0.77(0.28 \\
2.04)\end{array}$ & $\begin{array}{l}0.08(0.03, \\
0.22)\end{array}$ & TAF & $\begin{array}{l}0.47(0.06 \\
3.66)\end{array}$ \\
\hline $\begin{array}{l}0.69(0.42 \\
1.21)\end{array}$ & $\begin{array}{l}0.86(0.37 \text {, } \\
2)\end{array}$ & $\begin{array}{l}1.06(0.33 \\
3.81)\end{array}$ & $\begin{array}{l}1.29(0.75 \\
2.29)\end{array}$ & $\begin{array}{l}0.52(0.2, \\
1.39)\end{array}$ & $\begin{array}{l}0.72(0.37, \\
1.34)\end{array}$ & $\begin{array}{l}1.62(0.63, \\
3.84)\end{array}$ & $\begin{array}{l}2.08(0.73 \text {, } \\
5.65)\end{array}$ & $\begin{array}{l}0.93(0.25 \\
3.34)\end{array}$ & $\begin{array}{l}1.11(0.52 \\
2.28)\end{array}$ & $\begin{array}{l}0.12(0.05 \text {, } \\
0.25)\end{array}$ & $\begin{array}{l}1.44(0.75 \\
2.8)\end{array}$ & TDF \\
\hline
\end{tabular}

Treatments are reported in alphabetical order. Empty cells in the right upper triangles are due to lack of studies comparing the treatment pairs in the HBeAg-negative networks. Data are reported as an odds ratio $(95 \% \mathrm{CI})$. Odd ratios higher than 1 favor the column-defining treatment. Significant results are in italic.

Virologic response, undetectable HBV DNA levels; $A L T$ normalization, normalization of serum alanine aminotransferase levels; $A D V$ adefovir $10 \mathrm{mg}$ daily; $A D V 2$ adefovir $30 \mathrm{mg}$ daily; $E T V$ entecavir; $L A M$ lamivudine; $T B V$ telbivudine; $P L A$ placebo; $T A F$ tenofovir alafenamide; $T D F$ tenofovir disoproxil fumarate; ADVTBV, ETVTDF, LAMADV1,

LAMADV2, and LAMTBV code for different combinations of antiviral agents with full details in Appendix C 
Table 4 Relative effects on serological responses of all pairs of interventions in odds ratios (95\% credible intervals) as calculated from the network meta-analyses using random effects models

$\mathrm{HBeAg}$ seroconversion in the left lower triangle and $\mathrm{HBeAg}$ loss in the right upper triangle $\mathrm{HBeAg}$-positive population

\begin{tabular}{|c|c|c|c|c|c|c|c|c|c|c|c|c|c|}
\hline \multicolumn{14}{|c|}{ Between study heterogeneity HBeAg-loss: $0.12(0.01-0.49)$} \\
\hline ADV & $\begin{array}{l}1.17 \\
(0.63, \\
2.14)\end{array}$ & $\begin{array}{l}1.2(0.46, \\
3.15)\end{array}$ & $\begin{array}{l}1.03 \\
(0.56, \\
1.91)\end{array}$ & $\begin{array}{l}0.68(0.28, \\
1.7)\end{array}$ & $\begin{array}{l}0.99(0.53, \\
1.82)\end{array}$ & & $\begin{array}{l}0.98(0.41, \\
2.25)\end{array}$ & $\begin{array}{l}0.94(0.37 \\
2.33)\end{array}$ & $\begin{array}{l}0.53(0.15 \\
1.81)\end{array}$ & $\begin{array}{l}1.4(0.74 \\
2.69)\end{array}$ & $\begin{array}{l}0.38(0.2, \\
0.7)\end{array}$ & $\begin{array}{l}2.07 \\
(0.84, \\
5.15)\end{array}$ & $\begin{array}{l}1.74 \\
(0.88, \\
3.46) \\
\end{array}$ \\
\hline $\begin{array}{l}0.9(0.44, \\
1.85)\end{array}$ & ADV2 & $\begin{array}{l}1.02(0.34, \\
3.1)\end{array}$ & $\begin{array}{l}0.88 \\
(0.39 \\
1.98)\end{array}$ & $\begin{array}{l}0.58(0.21, \\
1.66)\end{array}$ & $\begin{array}{l}0.84(0.38 \\
1.85)\end{array}$ & & $\begin{array}{l}0.84(0.3 \\
2.21)\end{array}$ & $\begin{array}{l}0.81(0.28, \\
2.26)\end{array}$ & $\begin{array}{l}0.46(0.12 \\
1.7)\end{array}$ & $\begin{array}{l}1.19 \\
(0.53, \\
2.76)\end{array}$ & $\begin{array}{l}0.33 \\
(0.16 \\
0.64)\end{array}$ & $\begin{array}{l}1.76(0.6 \\
5.21)\end{array}$ & $\begin{array}{l}1.48 \\
(0.62, \\
3.62)\end{array}$ \\
\hline $\begin{array}{l}0.88 \\
(0.33 \\
2.36)\end{array}$ & $\begin{array}{l}0.98(0.3, \\
3.15)\end{array}$ & ADVTBV & $\begin{array}{l}0.85 \\
(0.32, \\
2.32)\end{array}$ & $\begin{array}{l}0.57(0.18, \\
1.86)\end{array}$ & $\begin{array}{l}0.82(0.31, \\
2.17)\end{array}$ & & $\begin{array}{l}0.81(0.26, \\
2.45)\end{array}$ & $\begin{array}{l}0.78(0.24, \\
2.58)\end{array}$ & $\begin{array}{l}0.44(0.11, \\
1.83)\end{array}$ & $\begin{array}{l}1.17 \\
(0.46, \\
3.01)\end{array}$ & $\begin{array}{l}0.32 \\
(0.11, \\
0.93)\end{array}$ & $\begin{array}{l}1.72(0.5, \\
5.96)\end{array}$ & $\begin{array}{l}1.44 \\
(0.49 \\
4.23) \\
\end{array}$ \\
\hline $\begin{array}{l}1.08 \\
(0.64, \\
1.89)\end{array}$ & $\begin{array}{l}1.2(0.53 \\
2.88)\end{array}$ & $\begin{array}{l}1.24(0.46, \\
3.44)\end{array}$ & ETV & $\begin{array}{l}0.66(0.35 \\
1.27)\end{array}$ & $\begin{array}{l}0.96(0.65, \\
1.41)\end{array}$ & & $\begin{array}{l}0.95(0.47, \\
1.89)\end{array}$ & $\begin{array}{l}0.91(0.41, \\
2.03)\end{array}$ & $\begin{array}{l}0.52(0.16 \\
1.56)\end{array}$ & $\begin{array}{l}1.35 \\
(0.83, \\
2.27)\end{array}$ & $\begin{array}{l}0.37 \\
(0.18, \\
0.74)\end{array}$ & $\begin{array}{l}2(0.88, \\
4.71)\end{array}$ & $\begin{array}{l}1.68 \\
(0.92, \\
3.17)\end{array}$ \\
\hline $\begin{array}{l}1.89 \\
(0.81, \\
4.53)\end{array}$ & $\begin{array}{l}2.09 \\
(0.72, \\
6.45)\end{array}$ & $\begin{array}{l}2.15(0.65, \\
7.38)\end{array}$ & $\begin{array}{l}1.74 \\
(0.88, \\
3.42) \\
\end{array}$ & ETVTDF & $\begin{array}{l}1.44(0.67, \\
3.05)\end{array}$ & & $\begin{array}{l}1.41(0.55, \\
3.64)\end{array}$ & $\begin{array}{l}1.38(0.5, \\
3.88)\end{array}$ & $\begin{array}{l}0.78(0.21, \\
2.88)\end{array}$ & $\begin{array}{l}2.04 \\
(0.91, \\
4.65)\end{array}$ & $\begin{array}{l}0.56 \\
(0.21, \\
1.45)\end{array}$ & $\begin{array}{l}3.03 \\
(1.04, \\
8.84) \\
\end{array}$ & $\begin{array}{l}2.54 \\
(1.04, \\
6.27)\end{array}$ \\
\hline $\begin{array}{l}1.12 \\
(0.66,1.9)\end{array}$ & $\begin{array}{l}1.24 \\
(0.54 \\
2.87) \\
\end{array}$ & $\begin{array}{l}1.27(0.49, \\
3.37)\end{array}$ & $\begin{array}{l}1.03(0.7 \\
1.47)\end{array}$ & $\begin{array}{l}0.59(0.27 \\
1.26)\end{array}$ & LAM & & $\begin{array}{l}0.99(0.55 \\
1.76)\end{array}$ & $\begin{array}{l}0.96(0.47, \\
1.91)\end{array}$ & $\begin{array}{l}0.54(0.18 \\
1.54)\end{array}$ & $\begin{array}{l}1.41 \\
(1.02, \\
2.04) \\
\end{array}$ & $\begin{array}{l}0.39(0.2, \\
0.75)\end{array}$ & $\begin{array}{l}2.09 \\
(0.87, \\
5.23) \\
\end{array}$ & $\begin{array}{l}1.76(0.9 \\
3.55)\end{array}$ \\
\hline $\begin{array}{l}0.37 \\
(0.15 \\
0.88)\end{array}$ & $\begin{array}{l}0.41 \\
(0.14, \\
1.25)\end{array}$ & $\begin{array}{l}0.42(0.12, \\
1.48)\end{array}$ & $\begin{array}{l}0.34 \\
(0.13, \\
0.84)\end{array}$ & $\begin{array}{l}0.2(0.06, \\
0.6)\end{array}$ & $\begin{array}{l}0.33(0.14, \\
0.78)\end{array}$ & LAN & & & & & & & \\
\hline $\begin{array}{l}1.33 \\
(0.59 \\
3.08) \\
\end{array}$ & $\begin{array}{l}1.48 \\
(0.52,4.3)\end{array}$ & $\begin{array}{l}1.51(0.48, \\
4.83)\end{array}$ & $\begin{array}{l}1.22 \\
(0.59 \\
2.56) \\
\end{array}$ & $\begin{array}{l}0.7(0.26, \\
1.89)\end{array}$ & $\begin{array}{l}1.19(0.63, \\
2.28)\end{array}$ & $\begin{array}{l}3.59(1.22 \\
10.74)\end{array}$ & LAMADV1 & $\begin{array}{l}0.97(0.48, \\
1.92)\end{array}$ & $\begin{array}{l}0.55(0.16 \\
1.86)\end{array}$ & $\begin{array}{l}1.43 \\
(0.74, \\
2.86) \\
\end{array}$ & $\begin{array}{l}0.39 \\
(0.16, \\
0.98)\end{array}$ & $\begin{array}{l}2.11 \\
(0.74, \\
6.28) \\
\end{array}$ & $\begin{array}{l}1.77 \\
(0.74, \\
4.43) \\
\end{array}$ \\
\hline $\begin{array}{l}1.5(0.6, \\
3.92)\end{array}$ & $\begin{array}{l}1.67 \\
(0.55, \\
5.18) \\
\end{array}$ & $\begin{array}{l}1.71(0.5 \\
6.13)\end{array}$ & $\begin{array}{l}1.37 \\
(0.59 \\
3.33) \\
\end{array}$ & $\begin{array}{l}0.79(0.27 \\
2.39)\end{array}$ & $\begin{array}{l}1.34(0.63, \\
2.99)\end{array}$ & $\begin{array}{l}4.04(1.31, \\
13.28)\end{array}$ & $\begin{array}{l}1.13(0.51, \\
2.5)\end{array}$ & LAMADV2 & $\begin{array}{l}0.57(0.15 \\
2.07)\end{array}$ & $\begin{array}{l}1.48 \\
(0.69, \\
3.29) \\
\end{array}$ & $\begin{array}{l}0.4(0.16 \\
1.09)\end{array}$ & $\begin{array}{l}2.19 \\
(0.71 \\
6.98) \\
\end{array}$ & $\begin{array}{l}1.85 \\
(0.71, \\
4.97) \\
\end{array}$ \\
\hline $\begin{array}{l}2.14 \\
(0.63, \\
7.41)\end{array}$ & $\begin{array}{l}2.4(0.59 \\
9.44)\end{array}$ & $\begin{array}{l}2.39(0.59 \\
10.72)\end{array}$ & $\begin{array}{l}1.95 \\
(0.61, \\
6.39)\end{array}$ & $\begin{array}{l}1.14(0.29 \\
4.36)\end{array}$ & $\begin{array}{l}1.9(0.63 \\
6.02)\end{array}$ & $\begin{array}{l}5.68(1.43 \\
24.18)\end{array}$ & $\begin{array}{l}1.6(0.44 \\
5.86)\end{array}$ & $\begin{array}{l}1.42(0.37, \\
5.5)\end{array}$ & LAMTBV & $\begin{array}{l}2.6(0.93 \\
7.78)\end{array}$ & $\begin{array}{l}0.72(0.2, \\
2.56)\end{array}$ & $\begin{array}{l}3.85 \\
(0.99 \\
15.89) \\
\end{array}$ & $\begin{array}{l}3.25 \\
(0.93, \\
11.81)\end{array}$ \\
\hline $\begin{array}{l}0.79 \\
(0.43, \\
1.41)\end{array}$ & $\begin{array}{l}0.87 \\
(0.36,2.1)\end{array}$ & $\begin{array}{l}0.89(0.35, \\
2.3)\end{array}$ & $\begin{array}{l}0.72 \\
(0.42, \\
1.16)\end{array}$ & $\begin{array}{l}0.42(0.17 \\
0.94)\end{array}$ & $\begin{array}{l}0.7(0.48, \\
0.99)\end{array}$ & $\begin{array}{l}2.1(0.84, \\
5.33)\end{array}$ & $\begin{array}{l}0.59(0.28 \\
1.2)\end{array}$ & $\begin{array}{l}0.53(0.22 \\
1.21)\end{array}$ & $\begin{array}{l}0.37(0.12 \\
1.07)\end{array}$ & TBV & $\begin{array}{l}0.27 \\
(0.13, \\
0.55)\end{array}$ & $\begin{array}{l}1.48 \\
(0.58, \\
3.82) \\
\end{array}$ & $\begin{array}{l}1.24 \\
(0.59 \\
2.61) \\
\end{array}$ \\
\hline $\begin{array}{l}2.98 \\
(1.54 \\
6.09)\end{array}$ & $\begin{array}{l}3.3(1.47 \\
7.83)\end{array}$ & $\begin{array}{l}3.38(1.16 \\
10.52)\end{array}$ & $\begin{array}{l}2.73 \\
(1.38, \\
5.61)\end{array}$ & $\begin{array}{l}1.57(0.6 \\
4.19)\end{array}$ & $\begin{array}{l}2.66(1.43, \\
5.27)\end{array}$ & $\begin{array}{l}8.02(2.91, \\
23.3)\end{array}$ & $\begin{array}{l}2.23(0.91 \\
5.82)\end{array}$ & $\begin{array}{l}1.98(0.71, \\
5.52)\end{array}$ & $\begin{array}{l}1.4(0.39 \\
4.99)\end{array}$ & $\begin{array}{l}3.77 \\
(1.89 \\
8.08)\end{array}$ & PLA & $\begin{array}{l}5.47 \\
(1.96, \\
15.07)\end{array}$ & $\begin{array}{l}4.57 \\
(1.98 \\
10.51)\end{array}$ \\
\hline $\begin{array}{l}0.56 \\
(0.24, \\
1.27)\end{array}$ & $\begin{array}{l}0.62 \\
(0.21, \\
1.85)\end{array}$ & $\begin{array}{l}0.63(0.19, \\
2.21)\end{array}$ & $\begin{array}{l}0.52 \\
(0.22, \\
1.16)\end{array}$ & $\begin{array}{l}0.3(0.1, \\
0.86)\end{array}$ & $\begin{array}{l}0.5(0.21 \\
1.19)\end{array}$ & $\begin{array}{l}1.5(0.48, \\
4.92)\end{array}$ & $\begin{array}{l}0.42(0.14 \\
1.23)\end{array}$ & $\begin{array}{l}0.37(0.11, \\
1.19)\end{array}$ & $\begin{array}{l}0.26(0.06 \\
1.08)\end{array}$ & $\begin{array}{l}0.71 \\
(0.28, \\
1.84)\end{array}$ & $\begin{array}{l}0.19 \\
(0.07 \\
0.51) \\
\end{array}$ & TAF & $\begin{array}{l}0.84 \\
(0.47,1.5) \\
\end{array}$ \\
\hline $\begin{array}{l}0.73 \\
(0.43, \\
1.23) \\
\end{array}$ & $\begin{array}{l}0.81 \\
(0.34, \\
1.95) \\
\end{array}$ & $\begin{array}{l}0.83(0.29, \\
2.4)\end{array}$ & $\begin{array}{l}0.67 \\
(0.38, \\
1.14) \\
\end{array}$ & $\begin{array}{l}0.39(0.16 \\
0.91)\end{array}$ & $\begin{array}{l}0.66(0.36 \\
1.17)\end{array}$ & $\begin{array}{l}1.96(0.75 \\
5.21)\end{array}$ & $\begin{array}{l}0.55(0.23 \\
1.31)\end{array}$ & $\begin{array}{l}0.49(0.18 \\
1.27)\end{array}$ & $\begin{array}{l}0.34(0.1 \\
1.19)\end{array}$ & $\begin{array}{l}0.93 \\
(0.49, \\
1.84) \\
\end{array}$ & $\begin{array}{l}0.25 \\
(0.11, \\
0.53) \\
\end{array}$ & $\begin{array}{l}1.3(0.69, \\
2.52)\end{array}$ & TDF \\
\hline
\end{tabular}

HBsAg loss for HBeAg-positive population

\begin{tabular}{|c|c|c|c|c|c|c|c|c|c|}
\hline \multicolumn{10}{|c|}{ Between study heterogeneity HBsAg loss: $0.81(0.04-1.92)$} \\
\hline ADV & $\begin{array}{l}2.9(0.05, \\
271.54) \\
\end{array}$ & $\begin{array}{l}3.85(0.03, \\
640.68)\end{array}$ & $\begin{array}{l}1.85 \\
(0.01, \\
329.3)\end{array}$ & $\begin{array}{l}1.69(0, \\
1130.85)\end{array}$ & $\begin{array}{l}1.8(0, \\
1261.02)\end{array}$ & $\begin{array}{l}1.8(0.01 \\
494.34)\end{array}$ & $\begin{array}{l}0.66(0, \\
332.63)\end{array}$ & $\begin{array}{l}13.33(0.19 \\
2314.97)\end{array}$ & $\begin{array}{l}4.41(0.25, \\
178.38)\end{array}$ \\
\hline $\begin{array}{l}0.34(0, \\
22.06)\end{array}$ & ETV & $\begin{array}{l}1.31(0.11, \\
15.76)\end{array}$ & $\begin{array}{l}0.63 \\
(0.05 \\
7.79)\end{array}$ & $\begin{array}{l}0.58(0.01, \\
63.34)\end{array}$ & $\begin{array}{l}0.59(0, \\
65.36)\end{array}$ & $\begin{array}{l}0.61(0.02, \\
15.05)\end{array}$ & $\begin{array}{l}0.23(0, \\
15.45)\end{array}$ & $\begin{array}{l}4.53(0.07, \\
557.31)\end{array}$ & $\begin{array}{l}1.58(0.09, \\
30.77)\end{array}$ \\
\hline $\begin{array}{l}0.26(0, \\
32.13)\end{array}$ & $\begin{array}{l}0.76 \\
(0.06 \\
9.37) \\
\end{array}$ & ETVTDF & $\begin{array}{l}0.48 \\
(0.01, \\
17.23) \\
\end{array}$ & $\begin{array}{l}0.43(0, \\
89.87)\end{array}$ & $\begin{array}{l}0.45(0, \\
97.49)\end{array}$ & $\begin{array}{l}0.47(0.01, \\
26.73)\end{array}$ & $\begin{array}{l}0.17(0, \\
20.11)\end{array}$ & $\begin{array}{l}3.47(0.03, \\
718.78)\end{array}$ & $\begin{array}{l}1.18(0.03, \\
56.56)\end{array}$ \\
\hline $\begin{array}{l}0.54(0, \\
77.74)\end{array}$ & $\begin{array}{l}1.59 \\
(0.13 \\
20.71) \\
\end{array}$ & $\begin{array}{l}2.1(0.06 \\
75.21)\end{array}$ & LAM & $\begin{array}{l}0.93(0.02 \\
49.96)\end{array}$ & $\begin{array}{l}0.94(0.02, \\
52.48)\end{array}$ & $\begin{array}{l}0.98(0.13, \\
7.42)\end{array}$ & $\begin{array}{l}0.38(0.01, \\
9.99)\end{array}$ & $\begin{array}{l}7.24(0.06, \\
1694.37)\end{array}$ & $\begin{array}{l}2.53(0.05, \\
129.33)\end{array}$ \\
\hline $\begin{array}{l}0.59(0, \\
325.27)\end{array}$ & $\begin{array}{l}1.73 \\
(0.02 \\
198.06)\end{array}$ & $\begin{array}{l}2.3(0.01 \\
483.26)\end{array}$ & $\begin{array}{l}1.08 \\
(0.02, \\
61.42) \\
\end{array}$ & LAMADV1 & $\begin{array}{l}1.03(0.02, \\
61.82)\end{array}$ & $\begin{array}{l}1.05(0.01, \\
98.11)\end{array}$ & $\begin{array}{l}0.39(0, \\
76.6)\end{array}$ & $\begin{array}{l}8.2(0.01, \\
6590.4)\end{array}$ & $\begin{array}{l}2.86(0.01, \\
665.43)\end{array}$ \\
\hline $\begin{array}{l}0.56(0, \\
323.9)\end{array}$ & $\begin{array}{l}1.7(0.02, \\
206.83)\end{array}$ & $\begin{array}{l}2.23(0.01, \\
498.84)\end{array}$ & $\begin{array}{l}1.07 \\
(0.02, \\
62.58) \\
\end{array}$ & $\begin{array}{l}0.97(0.02 \\
61.37)\end{array}$ & LAMADV2 & $\begin{array}{l}1.01(0.01, \\
94.99)\end{array}$ & $\begin{array}{l}0.39(0, \\
76.92)\end{array}$ & $\begin{array}{l}7.85(0.01, \\
6778.66)\end{array}$ & $\begin{array}{l}2.7(0.01, \\
693.81)\end{array}$ \\
\hline $\begin{array}{l}0.55(0, \\
114.71)\end{array}$ & $\begin{array}{l}1.64 \\
(0.07, \\
44.14) \\
\end{array}$ & $\begin{array}{l}2.12(0.04, \\
136.67)\end{array}$ & $\begin{array}{l}1.02 \\
(0.13, \\
7.72)\end{array}$ & $\begin{array}{l}0.96(0.01, \\
88.89)\end{array}$ & $\begin{array}{l}0.99(0.01, \\
84.19)\end{array}$ & $\mathrm{TBV}$ & $\begin{array}{l}0.38(0, \\
17.85)\end{array}$ & $\begin{array}{l}7.4(0.04, \\
2548.22)\end{array}$ & $\begin{array}{l}2.61(0.03, \\
211.32)\end{array}$ \\
\hline $\begin{array}{l}1.5(0, \\
821.17)\end{array}$ & $\begin{array}{l}4.35 \\
(0.06, \\
468.91) \\
\end{array}$ & $\begin{array}{l}5.75(0.05, \\
1160.17) \\
\end{array}$ & $\begin{array}{l}2.67(0.1, \\
134.84)\end{array}$ & $\begin{array}{l}2.53(0.01, \\
655.63)\end{array}$ & $\begin{array}{l}2.57(0.01, \\
747.77)\end{array}$ & $\begin{array}{l}2.64(0.06, \\
208.83)\end{array}$ & PLA & $\begin{array}{l}20.47(0.05 \\
15819.77) \\
\end{array}$ & $\begin{array}{l}6.86(0.04, \\
1758.07)\end{array}$ \\
\hline $\begin{array}{l}0.08(0, \\
5.39)\end{array}$ & $\begin{array}{l}0.22(0, \\
15.21)\end{array}$ & $\begin{array}{l}0.29(0, \\
39.2)\end{array}$ & $\begin{array}{l}0.14(0, \\
18.17)\end{array}$ & $\begin{array}{l}0.12(0, \\
67.34)\end{array}$ & $\begin{array}{l}0.13(0, \\
67.65)\end{array}$ & $\begin{array}{l}0.14(0, \\
27.08)\end{array}$ & $\begin{array}{l}0.05(0, \\
18.71)\end{array}$ & TAF & $\begin{array}{l}0.37(0.01, \\
7.23)\end{array}$ \\
\hline $\begin{array}{l}0.23 \\
(0.01, \\
4.01)\end{array}$ & $\begin{array}{l}0.63 \\
(0.03, \\
11.07)\end{array}$ & $\begin{array}{l}0.84(0.02, \\
35.92)\end{array}$ & $\begin{array}{l}0.4(0.01, \\
18.37)\end{array}$ & $\begin{array}{l}0.35(0, \\
89.03)\end{array}$ & $\begin{array}{l}0.37(0, \\
87.88)\end{array}$ & $\begin{array}{l}0.38(0, \\
29.05)\end{array}$ & $\begin{array}{l}0.15(0, \\
23.22)\end{array}$ & $\begin{array}{l}2.67(0.14, \\
127.9)\end{array}$ & TDF \\
\hline
\end{tabular}

Treatments are reported in alphabetical order. Empty cells in the right upper triangle are due to lack of studies comparing the treatment pairs in the HBeAg loss network. Data are reported as an odds ratio $(95 \% \mathrm{CI})$. Odd ratios higher than 1 favor the column-defining treatment. Significant results are in italic.

HBeAg seroconversion hepatitis B e antigen seroconversion, HBeAg loss hepatitis $\mathrm{B}$ e antigen loss, $H B s A g$ loss hepatitis $\mathrm{B}$ surface antigen loss, $A D V$ adefovir 10 mg daily, $A D V 2$ adefovir $30 \mathrm{mg}$ daily, $E T V$ entecavir, $L A M$ lamivudine, $T B V$ telbivudine, $P L A$ placebo, $T A F$ tenofovir alafenamide, $T D F$ tenofovir disoproxil fumarate; ADVTBV, ETVTDF, LAMADV, LAMADV1, LAMADV2, and LAMTBV code for different combinations of antiviral agents with full details in Appendix C 


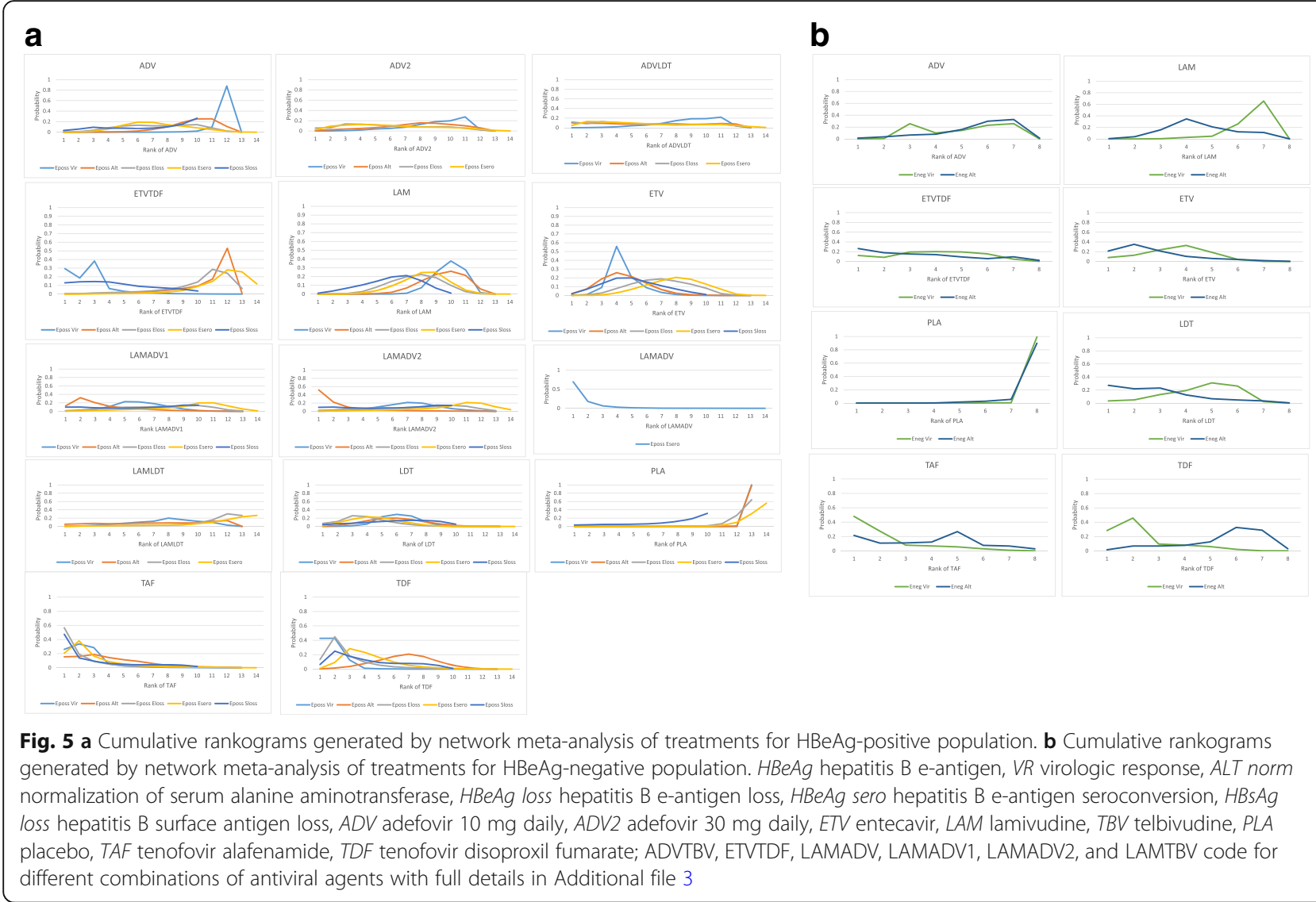

was significantly better than ETVTDF (OR $=0.3,95 \%$ CrI 0.1-0.86). LAMADV had the highest probability (69\%), followed by TAF (21\%) (Fig. 5a). For HBeAg loss (Table 4), TAF was significantly better than ETVTDF $(\mathrm{OR}=3.03,95 \% \mathrm{CrI} 1.04-8.84)$ and had the highest probability of achieving outcome (57\%) (Fig. 5a). With respect to HBsAg loss, no one drug significantly outperformed the others (Table 4).

\section{HBeAg negative patients: viral suppression and ALT normalization}

For virologic response, TAF and TDF had the highest probabilities of achieving viral suppression $(48 \%$ and $28 \%$ respectively) (Fig. 5b). However, pairwise comparisons across drugs (Table 3 ) did not show consistent superiority except comparing with ADV. For ALT normalization, TBV had the highest probability (27\%) of achieving this outcome (Fig. 5b). This was followed by ETVTDF (26\%) and TAF (22\%). However, pairwise comparisons across drugs (Table 3) did not show consistent superiority.

\section{Discussion}

We used network meta-analysis to integrate all available randomized trial evidence for the treatment of $\mathrm{CHB}$ by conducting direct and indirect comparisons among multiple treatment strategies. For each treatment within the Bayesian framework, we estimated the posterior probability of achieving a specific outcome and presented the results in the form of rankogram. However, we have to interpret this probability ranking with caution. The choice of the most desirable treatment should not be based solely on the ranking, but should take the relative effect into account [64].

Treatment recommendations depend not only on effectiveness but also on many other factors such as adverse events, potential for viral resistance, cost, patients' preference and values, and availability in the care setting. In addition, the choice has to be made across five relevant outcomes for $\mathrm{HBeAg}$-positive patients and two outcomes for HBeAg-negative patients. A treatment that is best in one outcome may not fare well in another outcome.

Across all outcomes and in both HBeAg-positive and HBeAg-negative populations, TAF emerged as the treatment with the most consistent performance (Fig. 5a, b). TAF was approved by FDA in 2016 for the treatment of $\mathrm{CHB}$. It is a prodrug of tenofovir and has a better safety profile with respect to renal function and bone mineral density compared to TDF [65-68]. It is rapidly converted 
to the active metabolite intracellularly. With reduced systemic exposure to the active metabolite, the off-target kidney and bone exposure are thereby reduced. It has the same resistance profile as TDF [69]. However, TAF is costly (estimated US\$15,570.90 per patient annually at US average wholesale price of $\$ 42.66 / 25 \mathrm{mg}$ tablet) [70], though one published study has suggested that TAF may nonetheless be cost effective [71].

Prior to 2017, TDF and ETV were the recommended oral drugs for $\mathrm{CHB}$ in the international guidelines because they have high effectiveness and a high barrier to resistance [72, 73]. With TDF, renal complications (nephropathy and Fanconi syndrome) and osteomalacia have been reported [74], but no resistance has been detected to date [7]. ETV has been associated with lactic acidosis and a low but slowly emerging resistance pattern $[74,75]$.

We found significant inconsistencies when studies with PEG-INF were included in the NMA with oral nucleos(t)ides. Among these studies, PEG-IFN was used in different dosages; the duration of treatment was shorter than the oral nucleos(t)ides, and PEG-INF was combined in different order with oral nucleos(t)ides in different combination therapies. This means that there were effect modifiers in the identified studies that suggested the evidence should not be combined. For the purpose of this NMA, the studies identified were too heterogeneous to combine, which manifested as inconsistency in the network. On the basis of this clinical heterogeneity, we decided to further exclude studies that included the PEGIFN treatment.

Pegylated interferon- $\alpha$ (PEG-IFN) is a synthetic cytokine and is believed to act on the cell-mediated immunity [76]. PEG-IFN treatment has the benefit of finite treatment duration, a higher rate of $\mathrm{HBeAg}$ and $\mathrm{HBsAg}$ seroconversion, and no drug resistance [77]. However, it is associated with more adverse events (flu-like symptoms, neutropenia, anemia, thrombocytopenia, depression, neuropathy, and dermatological side effects) than any of the oral drugs [78]. The need for parenteral administration also adds to the low preference and compliance. For all these reasons, PEG-IFN is hardly used in clinical practice nowadays. It has also been shown that extended nucleos $(\mathrm{t})$ ide therapy could result in $\mathrm{HBsAg}$ loss (functional cure) rates similar to those reported with PEG-IFN therapy [79]. In recent years, using regression analysis, clinicians are able to identify and select specific patients with positive predictors for sustained response to PEG-IFN and reduce unnecessary exposure for patients who are not likely to respond [80, 81]. Certain combination strategies between a nucleos(t)ide and PEG-IFN have been shown to exhibit synergistic therapeutic effect resulting in greater viral suppression and higher rates of HBeAg loss and HBsAg loss [82, 83].
In this review, we included all eligible RCTs published prior to the end of 2017. Our results are consonant with current clinical guidelines $[2,72,84]$ which recommend TAF, TDF, and ETV to be the first-line treatment for $\mathrm{CHB}$, and PEG-IFN for selective patient groups. These recommendations are based on evidence reviews, consensus of expert panels, and ratings on the Grading of Recommendations, Assessment, Development and Evaluation system. Our NMA provides comprehensive comparative evidence for these treatment recommendations.

Our analysis is also broadly consonant with other evidence reviews. In comparison with the NMAs published in 2010 and 2015 [9, 85], this analysis has included a new drug, TAF, and many new combination strategies. There are some differences in ranking. All three NMAs ranked TDF first for virologic suppression for HBeAgpositive patients. For ALT normalization, the two previous NMAs ranked TDF first for HBeAg-positive patients and TBV first for HBeAg-negative patients. We ranked TAF first for HBeAg-positive patients and TBV first for HBeAg-negative patients.

Our study is in agreement with the findings of another two NMAs in that TBV was superior to most other nucleos(t)ides in terms of HBeAg loss and seroconversion $[86,87]$. This was until the arrival of TAF which superseded TBV.

Since our previous review in 2010, drug combinations have been investigated increasingly to delay the emergence of resistance, to treat patients with previous treatment failure $[88,89]$, and most importantly to increase immune control over the virus, as indicated by $\mathrm{HBeAg}$ loss and HBsAg loss [38, 44]. However, there are relatively few studies at present, and sample sizes are small.

Our study has a few limitations that merit discussion. Firstly, we included studies that spanned 19 years. The laboratory methods used for quantification of HBV DNA have evolved tremendously in the last decade with detection threshold as low as $<10 \mathrm{IU} / \mathrm{ml}$ (approximately 50 copies $/ \mathrm{ml}$ ). Consequently, the definition for virologic response varied widely between older studies and recent studies and ranged from $<20,000 \mathrm{IU} / \mathrm{ml} \quad(100,000$ copies $/ \mathrm{ml})$ to $<15 \mathrm{IU} / \mathrm{ml}(75 \mathrm{copies} / \mathrm{ml})$. Even though we only included in our analysis those outcome data with detection limits of $\leq 200 \mathrm{IU} / \mathrm{ml}$ (1000 copies/ml), this is still an important source of variability. Secondly, in order to include as many interventions as possible to inform the network, we did not specify participants' baseline characteristics in our eligibility criteria. This could be an important source of heterogeneity. However, we tried to reduce baseline variability by excluding studies with decompensated cirrhosis, hepatocellular carcinoma, and liver transplant cohorts. Additional file 4 showed the variation in age, gender, baseline HBV DNA 
level, and baseline ALT level among the included studies. Studies with more favorable patient selection criteria are expected to produce more favorable results. Viral suppression is highly dependent on the baseline HBVDNA levels and treatment duration. It has been shown that baseline HBV DNA and HBsAg levels are strong predictors of virologic response to different $\mathrm{CHB}$ treatments [90-92]. ALT normalization is highly dependent on the inclusion criteria of ALT level and the presence of concomitant disease such as nonalcoholic steatohepatitis. Other baseline variables such as HBV genotypes also have correlation with treatment outcome $[93,94]$. This variation in baseline clinical characteristics is a recognized source of heterogeneity in NMA $[95,96]$. Thirdly, the small number of studies and patients limited the ability to conduct pairwise comparisons. This is particularly true for combination therapies. These small sample sizes produced wide credible intervals and reduced the number of closed loops in the network, especially for the HBeAg-negative population. This is also true for outcomes with rare events such as HBsAg loss, where the observed wide credible intervals indicate large uncertainty in differences between treatments. Fourthly, for some studies where information comes from fewer studies and more rare events, the estimate of betweenstudy heterogeneity is more heavily dependent on the choice of the (vague) prior. As a consequence, the estimate of uncertainty for some of the relative effectiveness parameters is inflated. The mean estimates or relative effects, however, have remained unchanged. Lastly, given that our review was limited to studies written in English, useful studies in other languages may have been missed.

\section{Conclusions}

The currently available treatment options are either nucleos(t)ide-based or interferon-based or their combinations. For both hepatitis B e antigen-positive and -negative populations, tenofovir disoproxil fumarate and tenofovir alafenamide are the most effective agent for virologic suppression. Our NMA confirmed the low probabilities and overall difficulty of achieving such endpoints as HBeAg loss/seroconversion or HBsAg loss. Even when virologic suppression is achieved, relapse rate is high and long-term treatment is necessary. Research for new treatment strategies and targets is necessary to achieve a functional cure. Currently, many new potential drugs targeting directly at the genomic viral reservoirs (cccDNA and integrated viral DNA) and agents boosting innate and $\mathrm{HBV}$-specific immunity are in the pipelines [97-100]. Until these new therapies arrive, best practice for care of HBV patients should be based on current evidence, as reported here.

\section{Additional files}

Additional file 1: PRISMA flow diagram and full search strategy for Ovid MEDLINE. (PDF 623 kb)

Additional file 2: Cochrane risk of bias assessment results. (PDF $375 \mathrm{~kb}$ ) Additional file 3: Characteristics of included randomized controlled trials. (PDF $358 \mathrm{~kb}$ )

Additional file 4: Participant baseline characteristics of included randomized controlled trials. (PDF 398 kb)

Additional file 5: Network inconsistency evaluation. (PDF 59 kb)

Additional file 6: Relative effects on outcomes of all pairs of interventions in Odds Ratios ( $95 \%$ credible intervals) as calculated from the network meta-analyses using fixed effects models. (PDF $122 \mathrm{~kb}$ )

\section{Abbreviations}

ADV: Adefovir; ALT: Alanine transaminase; CHB: Chronic hepatitis B; ETV: Entecavir; HBeAg: Hepatitis B e antigen; HBsAg: Hepatitis B surface antigen; HBV: Hepatitis B virus; HCC: Hepatocellular carcinoma;

LAM: Lamivudine; NMA: Network meta-analysis; PEG-IFN: Pegylated interferon alfa; RCT: Randomized controlled trial; TAF: Tenofovir alafenamide;

TBV: Telbivudine; TDF: Tenofovir disoproxil fumarate

\section{Acknowledgements}

The authors would like to thank The Ontario Drug Policy Research Network for supporting this project.

\section{Authors' contributions}

WW, PP, and AH performed the network meta-analysis and interpreted the results. JB designed and conducted the literature search. JW, AE, YS, AP, MT, $M Y$, and NR are independent reviewers responsible for screening, quality assessment, and data extraction. VR, HJ, and MK are clinical experts providing inputs for endpoint selection and clinical interpretation of results. WW, JW, and MK are major contributors in writing the manuscript. All authors read and approved the final manuscript.

\section{Funding}

This study was funded by The Ontario Drug Policy Research Network. Dr. Wong was supported by an Ontario Ministry of Research, Innovation, and Science Early Researcher Award.

\section{Availability of data and materials}

The datasets used and/or analyzed in this study are available from the corresponding author on reasonable request.

\section{Competing interests}

William Wong and Murray Krahn have received research support from the Canadian Liver Foundation. Harry L. A. Janssen received grants from AbbVie, Bristol Myers Squibb, Gilead Sciences, Innogenetics, Janssen, Medimmune, Medtronic, Merck, and Roche and is consultant for AbbVie, Benitec, Bristol Myers Squibb, Gilead Sciences, Janssen, Medimmune, Merck, Roche and Arbutus. None of the other co-authors has any conflict of interest to declare.

\section{Author details}

${ }^{1}$ Toronto Health Economics and Technology Assessment Collaborative (THETA), University of Toronto and University Health Network, Toronto, ON, Canada. 'University of Waterloo, School of Pharmacy, Waterloo, ON, Canada. ${ }^{3}$ University of Toronto, Leslie Dan Faculty of Pharmacy, Toronto, ON, Canada. ${ }^{4}$ The Hospital for Sick Children, Toronto, ON, Canada. ${ }^{5}$ The Ontario Drug Policy Research Network, St. Michael's Hospital, Toronto, ON, Canada. ${ }^{6}$ BioPharma Services Inc, Toronto, ON, Canada. ${ }^{7}$ Toronto Centre for Liver Disease, University Health Network, Toronto, ON, Canada. ${ }^{8}$ Ted Rogers Centre for Heart Research at Peter Munk Cardiac Centre, Toronto General Hospital Research Institute (TGHRI), University Health Network (UHN), Toronto, Canada. 'Institute of Health Policy, Management and Evaluation (IHPME), Dalla Lana School of Public Health, University of Toronto, Toronto, Canada.

${ }^{10}$ Diabetes Action Canada, CIHR SPOR Network, Toronto, Canada. 
Received: 14 December 2018 Accepted: 4 August 2019 Published online: 19 August 2019

\section{References}

1. Organization WH. Global hepatitis report 2017: World Health Organization; 2017.

2. Liver EAFTSOT. EASL 2017 Clinical practice guidelines on the management of hepatitis B virus infection. J Hepatol. 2017;67(2):370-98.

3. Sherman M, Shafran S, Burak K, Doucette K, Wong W, Girgrah N, et al. Management of chronic hepatitis B: consensus guidelines. Can J Gastroenterol. 2007;21 Suppl C:5C-24C.

4. Ghany MG, Doo EC. Antiviral Resistance and hepatitis B therapy. Hepatology (Baltimore, Md). 2009:49(5 Suppl):S174-S84.

5. Drugs@FDA: FDA Approved Drug Products: Tenofovir Alafenamide Fumarate [Available from: https://www.accessdata.fda.gov/scripts/cder/daf/ index.cfm?event=overview.process\&ApplNo=208464. Accessed 1 July 2018.

6. Health Canada Drug Product Database [Available from: https://www.canada. ca/en/health-canada/services/drugs-health-products/drug-products/drugproduct-database.html. Accessed 1 July 2018.

7. Liu Y, Corsa A, Buti M, Cathcart A, Flaherty J, Miller M, et al. No detectable resistance to tenofovir disoproxil fumarate in $\mathrm{HBeAg}+$ and $\mathrm{HBeAg}$ - patients with chronic hepatitis B after 8 years of treatment. Journal of viral hepatitis. 2017;24(1):68-74.

8. Sherman M, Lee SS. Canadian patients with chronic hepatitis B cannot access appropriate drug treatments: a call for change. Can J Gastroenterol. 2011;25(10):538-41.

9. Woo G, Tomlinson G, Nishikawa Y, Kowgier M, Sherman M, Wong DKH, et al. Tenofovir and entecavir are the most effective antiviral agents for chronic hepatitis B: a systematic review and Bayesian meta-analyses. Gastroenterology. 2010;139(4):1218-29.

10. Ismail S, Hafez HA, Darweesh SK, Kamal KH, Esmat G. Virologic response and breakthrough in chronic hepatitis B Egyptian patients receiving lamivudine therapy. Ann Gastroenterol. 2014;27(4):380-6.

11. Huang Y-J, Chang C-S, Peng Y-C, Yeh H-Z, Yang S-S. On-treatment HBV DNA dynamics predict virological breakthrough in entecavir-treated HBeAgpositive chronic hepatitis B. PLoS One. 2017;12(3):e0174046

12. Ustianowski A, Arends JE. Tenofovir: What we have learnt after 7.5 Million person-years of use. infectious diseases and therapy. 2015;4(2):145-57.

13. Wong WW, Pechlivanoglou P, Erman A, Saeed Y, Younis M, Rayad NZ, et al. FINAL CENSORED REPORT: Treatment for chronic hepatitis B. 2015.

14. Liberati A, Altman DG, Tetzlaff J, Mulrow C, Gotzsche PC, loannidis JP, et al. The PRISMA statement for reporting systematic reviews and meta-analyses of studies that evaluate healthcare interventions: explanation and elaboration. BMJ. 2009;339:b2700.

15. Moher D, Shamseer L, Clarke M, Ghersi D, Liberati A, Petticrew M, et al. Preferred reporting items for systematic review and meta-analysis protocols (PRISMA-P) 2015 statement. Syst Rev. 2015;4:1

16. Higgins JPT, Green S. Cochrane handbook for systematic reviews of interventions version 5.1.0 The Cochrane Collaboration; [updated March 2011]. Available from: http://handbook-5-1.cochrane.org/.

17. Higgins JPT, Altman DG, Gøtzsche PC, Jüni P, Moher D, Oxman AD, et al. The Cochrane Collaboration's tool for assessing risk of bias in randomised trials. BMJ. 2011;343.

18. Plummer M. JAGS: a program for analysis of Bayesian graphical models using Gibbs sampling. Vienna: Proceedings of the 3rd international workshop on distributed statistical computing; 2003.

19. van Valkenhoef $G$, Lu G, de Brock B, Hillege H, Ades AE, Welton NJ. Automating network meta-analysis. Res Synth Methods. 2012;3(4):285-99.

20. Brooks SP, Gelman A. General methods for monitoring convergence of iterative simulations. Journal of computational and graphical statistics. 1998; 7(4):434-55

21. Dias $S$, Welton N, Caldwell D, Ades A. Checking consistency in mixed treatment comparison meta analysis. Statistics in medicine. 2010;29(7-8): 932-44.

22. Brouwer WP, Xie Q, Sonneveld MJ, Zhang N, Zhang Q, Tabak F, et al. Adding pegylated interferon to entecavir for hepatitis B e antigen-positive chronic hepatitis B: A multicenter randomized trial (ARES study). Hepatology. 2015;61(5):1512-22.

23. Cao ZH, Ma LN, Zhang HW, Liu YL, Chen XY. Extended treatment with peginterferon alpha-2a in combination with lamivudine or adefovir for 96 weeks yields high rates of HBeAg and HBsAg seroconversion. Journal of Digestive Diseases. 2013;14:446-50.

24. Chan $\mathrm{HL}$, Heathcote EJ, Marcellin P, Lai CL, Cho M, Moon YM, et al. Treatment of hepatitis $B$ e antigen positive chronic hepatitis with telbivudine or adefovir: a randomized trial. Ann Intern Med. 2007; 147(11):745-54.

25. Chan HL, Fung S, Seto WK, Chuang W-L, Chen C-Y, Kim HJ, et al. Tenofovir alafenamide versus tenofovir disoproxil fumarate for the treatment of HBeAg-positive chronic hepatitis B virus infection: a randomised, doubleblind, phase 3, non-inferiority trial. The Lancet Gastroenterology \& Hepatology. 2016;1(3):185-95.

26. Chang TT, Gish RG, de Man R, Gadano A, Sollano J, Chao YC, et al. A comparison of entecavir and lamivudine for $\mathrm{HBeAg}$-positive chronic hepatitis B. N Engl J Med. 2006;354(10):1001-10.

27. Dienstag JL, Schiff ER, Wright TL, Perrillo RP, Hann HW, Goodman Z, et al. Lamivudine as initial treatment for chronic hepatitis B in the United States. N Engl J Med. 1999;341(17):1256-63.

28. He Z, Wang J, Liu K, Huang H, Du Y, Lin Z, et al. Randomized trial of lamivudine, adefovir, and the combination in HBeAg-positive chronic hepatitis B. Clinics and research in hepatology and gastroenterology [Internet]. 2012; 36(6):[592-597 pp.]. Available from: http://onlinelibrary.wiley. com/o/cochrane/clcentral/articles/488/CN-00859488/frame.html.

29. Janssen $H L$, van Zonneveld M, Senturk H, Zeuzem S, Akarca US, Cakaloglu Y, et al. Pegylated interferon alfa-2b alone or in combination with lamivudine for HBeAg-positive chronic hepatitis B: a randomised trial. Lancet. 2005; 365(9454):123-9.

30. Koike K, Suyama K, Ito H, Itoh H, Sugiura W. Randomized prospective study showing the non-inferiority of tenofovir to entecavir in treatment-naive chronic hepatitis B patients. Hepatology Research. 2017.

31. Lai CL, Chien RN, Leung NW, Chang TT, Guan R, Tai DI, et al. A one-year trial of lamivudine for chronic hepatitis B. Asia Hepatitis Lamivudine Study Group. N Engl J Med. 1998;339(2):61-8.

32. Lai CL, Leung $\mathrm{N}$, Teo EK, Tong M, Wong F, Hann HW, et al. A 1-year trial of telbivudine, lamivudine, and the combination in patients with hepatitis B e antigen-positive chronic hepatitis B. Gastroenterology. 2005;129(2):528-36.

33. Lau GK, Piratvisuth T, Luo KX, Marcellin P, Thongsawat S, Cooksley G, et al. Peginterferon Alfa-2a, lamivudine, and the combination for HBeAg-positive chronic hepatitis B. N Engl J Med. 2005:352(26):2682-95.

34. Leung N, Peng CY, Hann HW, Sollano J, Lao-Tan J, Hsu CW, et al. Early hepatitis $B$ virus DNA reduction in hepatitis $B$ e antigen-positive patients with chronic hepatitis B: A randomized international study of entecavir versus adefovir. Hepatology. 2009;49:72-9.

35. Liang X, Cheng J, Sun Y, Chen X, Li T, Wang H, et al. Randomized, three-arm study to optimize lamivudine efficacy in hepatitis B e antigen-positive chronic hepatitis B patients. Journal of gastroenterology and hepatology. 2015;30(4):748-55

36. Liu YH, Wu T, Sun N, Wang GL, Yuan JZ, Dai YR, et al. Combination therapy with pegylated interferon alpha-2b and adefovir dipivoxil in HBeAg-positive chronic hepatitis B versus interferon alone: a prospective, randomized study. Journal of Huazhong University of Science and Technology Medical Sciences. 2014:34:542-7.

37. Marcellin P, Chang TT, Lim SG, Tong MJ, Sievert W, Shiffman ML, et al. Adefovir dipivoxil for the treatment of hepatitis B e antigen-positive chronic hepatitis B. N Engl J Med. 2003;348(9):808-16.

38. Marcellin $\mathrm{P}$, Ahn $\mathrm{SH}$, Ma X, Caruntu FA, Tak WY, Elkashab M, et al. Combination of tenofovir disoproxil fumarate and peginterferon $a-2 a$ increases loss of hepatitis B surface antigen in patients with chronic hepatitis B. Gastroenterology. 2016;150(1):134-44. e10.

39. Ren FY, Piao DM, Piao XX. A one-year trial of entecavir treatment in patients with HBeAg-positive chronic hepatitis B. World J Gastroenterol. 2007;13(31):4264-7.

40. Sriprayoon T, Mahidol C, Ungtrakul T, Chun-on P, Soonklang K, Pongpun W, et al. Efficacy and safety of entecavir versus tenofovir treatment in chronic hepatitis B patients: A randomized controlled trial. Hepatology Research. 2017;47(3):E161-E168.

41. Sung JJY, Lai JY, Zeuzem S, Chow WC, Heathcote EJ, Perrillo RP, et al. Lamivudine compared with lamivudine and adefovir dipivoxil for the treatment of HBeAg-positive chronic hepatitis B. Journal of Hepatology. 2008;48(5):728-35.

42. Tseng K-C, Chen C-Y, Tsai H-W, Chang T-T, Chuang W-L, Hsu P-I, et al. Efficacy of entecavir in chronic hepatitis B patients with persistently normal 
alanine aminotransferase: randomized, double-blind, placebo-controlled study. Antivir Ther. 2014;19:755-64.

43. Xie Q, Zhou H, Bai X, Wu S, Chen J-J, Sheng J, et al. A randomized, openlabel clinical study of combined pegylated Interferon Alfa-2a (40KD) and Entecavir treatment for Hepatitis B "e" Antigen-Positive Chronic Hepatitis B. Clinical infectious diseases. 2014;59(12):1714-23.

44. Zhang K, Cao H, Liang J, Shu X, Sun H, Li G, et al. CONSORT: Effects of adding adefovirdipivoxil to peginterferon alfa-2a at different time points on HBeAg-positivepatients: A prospective, randomized study. Medicine. 2016:95(31).

45. Bozkaya H, Yurdaydin C, Idilman R, Tuzun A, Cinar K, Erkan O, et al. Lamivudine treatment in $\mathrm{HBeAg}$-negative chronic hepatitis $B$ patients with low level viraemia. Antiviral Therapy. 2005:10:319-25.

46. Buti M, Gane E, Seto WK, Chan HL, Chuang W-L, Stepanova T, et al. Tenofovir alafenamide versus tenofovir disoproxil fumarate for the treatment of patients with $\mathrm{HBeAg}$-negative chronic hepatitis B virus infection: a randomised, double-blind, phase 3, non-inferiority trial. The Lancet Gastroenterology \& Hepatology. 2016;1(3):196-206.

47. Chan HLY, Wang H, Niu JQ, Chim AML, Sung JJY. Two-year lamivudine treatment for hepatitis B e antigen-negative chronic hepatitis B: a doubleblind, placebo-controlled trial. Antiviral Therapy. 2007;12:345-53.

48. Hadziyannis SJ, Tassopoulos NC, Heathcote EJ, Chang TT, Kitis G, Rizzetto M, et al. Adefovir dipivoxil for the treatment of hepatitis B e antigen-negative chronic hepatitis B. N Engl J Med. 2003;348(9):800-7.

49. Kaymakoglu S, Oguz D, Gur G, Gurel S, Tankurt E, Ersoz G, et al. Pegylated interferon Alfa-2b monotherapy and pegylated interferon Alfa-2b plus lamivudine combination therapy for patients with hepatitis $B$ virus $E$ antigen-negative chronic hepatitis B. Antimicrob Agents Chemother. 2007; 51(8):3020-2.

50. Lai C-L, Shouval D, Lok AS, Chang T-T, Cheinquer H, Goodman Z, et al. Entecavir versus Lamivudine for patients with $\mathrm{HBeAg-negative} \mathrm{chronic}$ hepatitis B. New England Journal of Medicine. 2006;354(10):1011-20.

51. Lampertico P, Vigano M, Di Costanzo GG, Sagnelli E, Fasano M, Di Marco $V$, et al. Randomised study comparing 48 and 96 weeks peginterferon alpha-2a therapy in genotype $D$ HBeAg-negative chronic hepatitis B. Gut. 2013;62:290-8.

52. Lee KS, Kweon Y-O, Um S-H, Kim B-H, Lim YS, Paik SW, et al. Efficacy and safety of entecavir versus lamivudine over 5 years of treatment: a randomized controlled trial in Korean patients with hepatitis B e antigen-negative chronic hepatitis B. Clinical and molecular hepatology. 2017;23(4):331.

53. Marcellin P, Lau GK, Bonino F, Farci P, Hadziyannis $S$, Jin $R$, et al. Peginterferon alfa-2a alone, lamivudine alone, and the two in combination in patients with HBeAg-negative chronic hepatitis B. N Engl J Med. 2004; 351(12):1206-17.

54. Papadopoulos VP, Chrysagis DN, Protopapas AN, Goulis IG, Dimitriadis GT, Mimidis KP. Peginterferon alfa-2b as monotherapy or in combination with lamivudine in patients with hbeag-negative chronic hepatitis b: a randomised study. Medical Science Monitor. 2009;15:CR56-61.

55. Piccolo P, Lenci I, Demelia L, Bandiera F, Piras MR, Antonucci G, et al. A randomized controlled trial of pegylated interferon-alpha2a plus adefovir dipivoxil for hepatitis B e antigen-negative chronic hepatitis B. Antivir Ther. 2009;14(8):1165-74.

56. Tangkijvanich $\mathrm{P}$, Chittmittraprap S, Poovorawan $\mathrm{K}$, Limothai U, Khlaiphuengsin A, Chuaypen $\mathrm{N}$, et al. A randomized clinical trial of peginterferon alpha-2b with or without entecavir in patients with $\mathrm{HBeAg}$ negative chronic hepatitis $B$ : Role of host and viral factors associated with treatment response. Journal of viral hepatitis. 2016;23(6):427-38.

57. Tassopoulos NC, Volpes R, Pastore G, Heathcote J, Buti M, Goldin RD, et al. Efficacy of lamivudine in patients with hepatitis B e antigen-negative/ hepatitis B virus DNA-positive (precore mutant) chronic hepatitis B. Lamivudine Precore Mutant Study Group. Hepatology. 1999;29:889-96.

58. Hou J, Gao Z, Xie Q, Zhang J, Sheng J, Cheng J, et al. Tenofovir disoproxil fumarate vs adefovir dipivoxil in Chinese patients with chronic hepatitis B after 48 weeks: a randomized controlled trial. Journal of viral hepatitis. 2015; 22(2):85-93.

59. Jia JD, Hou JL, Yin YK, Tan DM, Xu DZ, Niu JQ, et al. Two-year results of a randomized, phase III comparative trial of telbivudine versus lamivudine in Chinese patients. Hepatology International. 2014;8:72-82.

60. Liaw YF, Gane E, Leung N, Zeuzem S, Wang Y, Lai CL, et al. 2-Year GLOBE trial results: telbivudine Is superior to lamivudine in patients with chronic hepatitis B. Gastroenterology. 2009;136:486-95.
61. Lok AS, Trinh H, Carosi G, Akarca US, Gadano A, Habersetzer F, et al. Efficacy of entecavir with or without tenofovir disoproxil fumarate for nucleos(t)ide-nave patients with chronic hepatitis B. Gastroenterology. 2012;143:619-28.e1.

62. Marcellin P, Heathcote EJ, Buti M, Gane E, de Man RA, Krastev Z, et al. Tenofovir Disoproxil Fumarate versus Adefovir Dipivoxil for Chronic Hepatitis B. N Engl J Med. 2008;359(23):2442-55.

63. Yao GB, Chen CW, Lu WL, Ren H, Tan DM, Wang YM, et al. Efficacy and safety of entecavir compared to lamivudine in nucleoside-naive patients with chronic hepatitis B: a randomized double-blind trial in China. Hepatology International. 2007;1:365-72.

64. Mbuagbaw L, Rochwerg B, Jaeschke R, Heels-Andsell D, Alhazzani W, Thabane $L$, et al. Approaches to interpreting and choosing the best treatments in network meta-analyses. Systematic Reviews. 2017:6(1):79.

65. Chan HL-Y, Buti M, Flisiak R, Ryder S, Streinu-Cercel A, Flaherty JF, et al. IDDF2018-ABS-0107 Efficacy and safety of TENOFOVIR ALAFENAMIDE (TAF) at 96 weeks in chronic HBV (CHB) patients with risk factors for use of TENOFOVIR DISOPROXIL FUMARATE (TDF). Gut. 2018;67(Suppl 2):A97-A.

66. Agarwal K, Brunetto M, Seto WK, Lim Y-S, Fung S, Marcellin P, et al. 96\&\#x202f; weeks treatment of tenofovir alafenamide $<$ em $>v s</ e m>$. tenofovir disoproxil fumarate for hepatitis B virus infection. Journal of Hepatology. 2018;68(4):672-81.

67. Ogawa E, Furusyo N, Nguyen MH. Tenofovir alafenamide in the treatment of chronic hepatitis B: design, development, and place in therapy. Drug Design, Development and Therapy. 2017;11:3197-204.

68. DeJesus E, Haas B, Segal-Maurer S, Ramgopal MN, Mills A, Margot N, et al. Superior efficacy and improved renal and bone safety after switching from a tenofovir disoproxil fumarate- to a tenofovir alafenamide-based regimen through 96 weeks of treatment. AIDS Research and Human Retroviruses. 2018;34(4):337-42

69. Ray AS, Fordyce MW, Hitchcock MJM. Tenofovir alafenamide: A novel prodrug of tenofovir for the treatment of Human Immunodeficiency Virus. Antiviral Research. 2016;125:63-70.

70. Chon YE, Kim SU, Lee CK, Heo J, Kim JK, Yoon KT, et al. Partial virological response to entecavir in treatment-naive patients with chronic hepatitis $B$. Antiviral Therapy. 2011;16:469-77.

71. Dusheiko G, Lim J, Liou I, Tafazzoli A, Deniz B, Thibault S-LC, et al. Costeffectiveness analysis of first-line administration of tenofoviR alafenamide (TAF), a novel nucleotide Reverse transcriptase inhibitor (NRTI), for the management of chronic hepatitis B (CHB) In the United States (US). Value in health. New York: Elsevier Science INC; 2017. p. 10010-1710.

72. Terrault NA, Bzowej NH, Chang KM, Hwang JP, Jonas MM, Murad MH. AASLD guidelines for treatment of chronic hepatitis B. Hepatology. 2016; 63(1):261-83.

73. Organization WH. Guidelines for the Prevention Care and Treatment of Persons with Chronic Hepatitis B Infection: Mar-15: World Health Organization; 2015.

74. Smith M. Class Update: Antivirals for Hepatitis B; 2012.

75. Kayaaslan B, Guner R. Adverse effects of oral antiviral therapy in chronic hepatitis B. World Journal of Hepatology. 2017;9(5):227-41.

76. Woo ASJ, Kwok R, Ahmed T. Alpha-interferon treatment in hepatitis B. Annals of Translational Medicine. 2017;5(7):159.

77. Rijckborst V, Sonneveld MJ, Janssen H. chronic hepatitis B-anti-viral or immunomodulatory therapy? Alimentary pharmacology \& therapeutics. 2011;33(5):501-13.

78. Negro F. Adverse effects of drugs in the treatment of viral hepatitis. Best Practice \& Research Clinical Gastroenterology. 2010;24(2):183-92.

79. Dienstag JL. Benefits and risks of nucleoside analog therapy for hepatitis B. Hepatology. 2009;49(S5):S112-S21.

80. Buster EH, Hansen BE, Lau GK, Piratvisuth T, Zeuzem S, Steyerberg EW, et al. Factors that predict response of patients with hepatitis $B$ e antigen-Positive chronic hepatitis B to peginterferon-alfa. Gastroenterology. 2009;137(6):2002-9.

81. Arends P, Sonneveld MJ, Janssen HL. Hepatitis B virus treatment: which patients should be treated with interferon? Clinical Liver Disease. 2013;2(1): $18-20$.

82. Su T-H, Liu C-J. Combination therapy for chronic hepatitis B: current updates and perspectives. Gut and liver. 2017;11(5):590.

83. Chen S, Zhang W. HBeAg-positive chronic hepatitis B patients with prior long-time exposure to nucleos (t) ide analogues:"Switch-to" or "add-on" PegIFN alfa, that is the question. Journal of hepatology. 2015;62(1):239. 
84. Terrault NA, Lok AS, McMahon BJ, Chang KM, Hwang JP, Jonas MM, et al. Update on prevention, diagnosis, and treatment of chronic hepatitis B: AASLD 2018 hepatitis B guidance. Hepatology. 2018;67(4):1560-99.

85. Govan L, Wu O, Xin Y, Hutchinson SJ, Hawkins N. Comparative effectiveness of antiviral treatment for hepatitis B: A systematic review and Bayesian network meta-analysis. European Journal of Gastroenterology and Hepatology. 2015;27(8):882-94.

86. Liang X, Fan R, Sun J, Shaikh J, Taneja A, Gupta S, et al. Effect of telbivudine versus other nucleos (t) ide analogs on HBeAg seroconversion and other outcomes in patients with chronic hepatitis B: a network meta-analysis. Advances in therapy. 2016;33(4):519-31.

87. Xing T, Xu H, Cao L, Ye M. HBeAg seroconversion in HBeAg-positive chronic hepatitis B Patients receiving long-term nucleos(t)ide analog treatment: a systematic review and network meta-analysis. PLOS ONE. 2017;12(1): e0169444.

88. Zoulim F, Białkowska-Warzecha J, Diculescu MM, Goldis AE, Heyne R, Mach $T$, et al. Entecavir plus tenofovir combination therapy for chronic hepatitis $B$ in patients with previous nucleos (t) ide treatment failure. Hepatology international. 2016;10(5):779-88.

89. Lim Y-S. Management of Antiviral Resistance in Chronic Hepatitis B. Gut and Liver. 2017;11(2):189-95.

90. Lin C-L, Kao J-H. Hepatitis B viral factors and treatment responses in chronic hepatitis B. Journal of the Formosan Medical Association. 2013; 112(6):302-11.

91. Wang Y-C, Yang S-S, Su C-W, Wang Y-J, Lee K-C, Huo T-I, et al. Predictors of response to pegylated interferon in chronic hepatitis $\mathrm{B}$ : a real-world hospital-based analysis. Scientific Reports. 2016;6:29605.

92. Cho J-Y, Sohn W, Sinn D-H, Gwak G-Y, Paik Y-H, Choi MS, et al. Long-term real-world entecavir therapy in treatment-naïve hepatitis B patients: baseline hepatitis B virus DNA and hepatitis B surface antigen levels predict virologic response. The Korean journal of internal medicine. 2017;32(4):636.

93. Brunetto MR, Marcellin P, Cherubini B, Yurdaydin C, Farci P, Hadziyannis SJ, et al. Response to peginterferon alfa-2a (40KD) in HBeAg-negative CHB: ontreatment kinetics of HBsAg serum levels vary by HBV genotype. Journal of hepatology. 2013;59(6):1153-9.

94. Enomoto M, Tamori A, Nishiguchi S. Hepatitis B virus genotypes and response to antiviral therapy. Clinical laboratory. 2006;52(1-2):43-7.

95. Li T, Puhan MA, Vedula SS, Singh S, Dickersin K. Network meta-analysishighly attractive but more methodological research is needed. BMC medicine. 2011;9(1):79.

96. Mealing S, Ghement I, Hawkins N, Scott DA, Lescrauwaet B, Watt M, et al. The importance of baseline viral load when assessing relative efficacy in treatment-naive $\mathrm{HBeAg}$-positive chronic hepatitis B: a systematic review and network meta-analysis. Systematic reviews. 2014;3(1):21.

97. Soriano V, Barreiro P, Benitez L, Peña JM, de Mendoza C. New antivirals for the treatment of chronic hepatitis B. Expert opinion on investigational drugs. 2017;26(7):843-51.

98. Emery JS, Feld JJ. Treatment of hepatitis B virus with combination therapy now and in the future. Best Practice \& Research Clinical Gastroenterology. 2017:31(3):347-55.

99. Gane EJ. Future anti-HBV strategies. Liver International. 2017;37(S1):40-4.

100. Tseng T-C, Kao J-H. Elimination of hepatitis B: is it a mission possible? BMC medicine. 2017;15(1):53.

\section{Publisher's Note}

Springer Nature remains neutral with regard to jurisdictional claims in published maps and institutional affiliations.

Ready to submit your research? Choose BMC and benefit from:
- fast, convenient online submission
- thorough peer review by experienced researchers in your field
- rapid publication on acceptance
- support for research data, including large and complex data types
- gold Open Access which fosters wider collaboration and increased citations
- maximum visibility for your research: over 100M website views per year
At BMC, research is always in progress.
Learn more biomedcentral.com/submissions

